\title{
Superconducting instability of a non-centrosymmetric system
}

\author{
Dorota Grzybowska and Grzegorz Harańa \\ Department of Quantum Technologies, Faculty of Fundamental Problems of Technology, Wrocław University of Science \\ and Technology, Wybrzeże Wyspiańskiego 27, 50-370 Wrocław, Poland
}

Received 18 August 2016 / Received in final form 1 January 2017

Published online 15 March 2017

(C) The Author(s) 2017. This article is published with open access at Springerlink.com

\begin{abstract}
The Fermi gas approach to the weak-coupling superconductivity in the non-centrosymmetric systems lead to a conclusion of an approximately spin-orbit coupling independent critical temperature of the singlet states as well as the triplet states defined by the order parameter aligned with the antisymmetric spin-orbit coupling vector. We indicate that the above results follow from a simplified approximation of a density of states by a constant Fermi surface value. Such a scenario does not properly account for the spinsplit quasiparticle energy spectrum and reduces the spin-orbit coupling influence on superconductivity to the bare pair-breaking effect of a lifted spin degeneracy. Applying the tight-binding model, which captures the primary features of the spin-split energy band, i.e., its enhanced width and the spin-orbit coupling induced redistribution of the spectral weights in the density of states, we calculate the critical temperature of a non-centrosymmetric superconductor. We report a general tendency of the critical temperature to be suppressed by the antisymmetric spin-orbit coupling. We indicate that, the monotonic decrease of the critical temperature may be altered by the spin-orbit coupling induced van Hove singularities which, when driven to the Fermi level, generate maxima in the phase diagram. Extending our considerations to the intermediate-coupling superconductivity we point out that the spin-orbit coupling induced change of the critical temperature depends on the structure of the electronic energy band and both - the strength and symmetry of the pair potential. Finally, we discuss the mixed singlet-triplet state superconducting instability and establish conditions concerning the symmetry of the singlet and triplet counterparts as well as the range of the spin-orbit coupling energy which make such a phase transition possible.
\end{abstract}

\section{Introduction}

Since a discovery of superconductivity in $\mathrm{CePt}_{3} \mathrm{Si}[1]$ the identification of a superconducting groundstate of a system with no inversion center has become the primary issue for a growing number of the non-centrosymmetric superconductors [2]. Quantitatively, the lack of the inversion center in the elementary crystal lattice cell is manifested by the induced relativistic effect of the antisymmetric spin-orbit coupling which establishes a momentum-dependent spin quantization axis represented by the parity breaking gyroscopic vector, $\gamma(\boldsymbol{k})[3-5]$. Coupling of the spin and momentum degrees of freedom lifts the spin degeneracy of the energy eigenstates and leads to a formation of a spin-split quasiparticle energy spectrum consisting of two bands discriminated by their opposite $\boldsymbol{\gamma}(\boldsymbol{k})$-axis spin projections and separated in energy by $2|\gamma(\boldsymbol{k})|$. As a result, a developed superconducting state is formed by a superposition of the two-band spin-singlet and spin-triplet states $[3-5]$ determined by the singlet $\Delta_{s} e(\boldsymbol{k})$ and triplet $\Delta_{t} \boldsymbol{d}(\boldsymbol{k})$ order parameters. The onset of a stable non-centrosymmetric superconducting state, which is discerned among the symmetry allowed states by the highest

\footnotetext{
a e-mail: haran@pwr.edu.pl
}

critical temperature $T_{c}$, was discussed within the weakcoupling superconducting Fermi gas approach by Frigeri et al. [6] in correspondence to the experimental evidence of superconductivity in $\mathrm{CePt}_{3} \mathrm{Si}$ and $\mathrm{MnSi}$. Such an approximation ignores, however, the most direct consequence of the spin-orbit coupling exemplified by a modified spinsplit density of states which in the Fermi gas scenario of the weak-coupling superconductivity is replaced by a constant and spin-orbit coupling independent Fermi surface value. Accordingly, the effect of the broken inversion symmetry on the critical temperature is reduced to the pair-breaking effect of a lifted spin degeneracy which, due to the weak-coupling Fermi surface pairing constraint, becomes detrimental to the Cooper pairs with a nonzero spin projection onto the quantization $\gamma(\boldsymbol{k})$-axis and results in a severe suppression of the triplet superconducting states defined by the order parameter $\boldsymbol{d}(\boldsymbol{k})$ nonparallel to $\gamma(\boldsymbol{k})$. On the other hand, the unaltered density of states at the Fermi level leads to approximately spin-orbit coupling independent transition to the superconducting state of spin projection zero with respect to the $\gamma(\boldsymbol{k})$-axis. The lack of the antisymmetric spin-orbit coupling effect on the critical temperature is exhibited then by the triplet state defined by $\boldsymbol{d}(\boldsymbol{k})$ parallel to $\gamma(\boldsymbol{k})$ as well as the singlet state, for which Frigeri et al. [6] confirmed the previous 
result of Barzykin and Gor'kov [7]. We verify the above statements and formulate the weak-coupling approach to the superconducting instability of a non-centrosymmetric system accounting for the spin-split electron band structure $[8-13]$. In the following, we reexamine the issue of the onset of superconductivity within the tight-binding model and communicate a general tendency of the antisymmetric spin-orbit coupling to suppress the critical temperature with the exception of systems displaying the Fermi level singularities of the spin-split density of states, for which we report a possible rise of $T_{c}$. Extending our considerations to the intermediate-coupling superconductors we indicate a significantly reduced initial suppression of the mixed intraband and interband $\boldsymbol{d}(\boldsymbol{k}) \bigvee \boldsymbol{\gamma}(\boldsymbol{k})$ triplet states which for the weak spin-orbit splitting becomes comparable to that of the intraband $\boldsymbol{d}(\boldsymbol{k}) \| \boldsymbol{\gamma}(\boldsymbol{k})$ state. We conclude, that the direct influence of the spin-split energy band, exemplified by a modified density of states, is an important element of the antisymmetric spin-orbit coupling effect on superconductivity and cannot be neglected in a discussion of superconducting properties of the non-centrosymmetric systems. In the last part of the paper we discuss the phase transition to the mixed singlet-triplet states which emerge from the uncoupled singlet and triplet states due to the spin-orbit interaction. We find that the onset of the coupled singlet-triplet states is possible for the singlet state belonging to the identity representation of the system symmetry point group and the triplet state determined by $\boldsymbol{d}(\boldsymbol{k})$ of a nonzero projection onto the $\gamma(\boldsymbol{k})$-axis. We establish that the mixed singlet-triplet superconducting instability takes place for a very low range of magnitude of the spin-orbit coupling energy $E_{S O}$ for nearly degenerate singlet and triplet states and extends to the range of $E_{S O}$ limited by the existence of the uncoupled states when their critical temperatures significantly differ. Units $\hbar=k_{B}=1$ are used throughout the paper.

\section{Method}

We consider a non-centrosymmetric superconducting system defined by the Hamiltonian

$$
\begin{aligned}
\hat{H}= & \sum_{\boldsymbol{k}, \alpha}\left(\varepsilon_{\boldsymbol{k}}-\mu\right) \hat{a}_{\boldsymbol{k} \alpha}^{\dagger} \hat{a}_{\boldsymbol{k} \alpha}+\sum_{\boldsymbol{k}, \alpha, \beta} \gamma(\boldsymbol{k}) \cdot \boldsymbol{\sigma}_{\alpha \beta} \hat{a}_{\boldsymbol{k} \alpha}^{\dagger} \hat{a}_{\boldsymbol{k} \beta} \\
& +\frac{1}{2} \sum_{\boldsymbol{k}, \alpha, \beta}\left[\Delta_{\alpha \beta}(\boldsymbol{k}) \hat{a}_{\boldsymbol{k} \alpha}^{\dagger} \hat{a}_{-\boldsymbol{k} \beta}^{\dagger}+\Delta_{\alpha \beta}^{\dagger}(\boldsymbol{k}) \hat{a}_{-\boldsymbol{k} \alpha} \hat{a}_{\boldsymbol{k} \beta}\right]
\end{aligned}
$$

where $\hat{a}_{\boldsymbol{k} \alpha}^{\dagger}$ and $\hat{a}_{\boldsymbol{k} \alpha}$ operators create and annihilate particles in the energy band $\varepsilon_{\boldsymbol{k}}, \mu$ is the chemical potential, $\hat{\boldsymbol{\sigma}}=\left(\hat{\sigma}_{x}, \hat{\sigma}_{y}, \hat{\sigma}_{z}\right)$ is the $1 / 2$ spin operator, and the broken parity symmetry is represented by the antisymmetric spin-orbit coupling vector $\gamma(\boldsymbol{k})=-\gamma(-\boldsymbol{k})$. The Hamiltonian (1) is accomplished by the mean-field equation for the off-diagonal order parameter $\hat{\Delta}(\boldsymbol{k})$

$$
\Delta_{\alpha \beta}(\boldsymbol{k})=\sum_{\boldsymbol{k}^{\prime}, \gamma, \delta} V_{\alpha \beta \gamma \delta}\left(\boldsymbol{k}, \boldsymbol{k}^{\prime}\right) \frac{\operatorname{Tr}\left\{e^{-\hat{H} / T} \hat{a}_{\boldsymbol{k}^{\prime} \gamma} \hat{a}_{-\boldsymbol{k}^{\prime} \delta}\right\}}{\operatorname{Tr} e^{-\hat{H} / T}}
$$

where $\operatorname{Tr}$ denotes a trace in the grand canonical ensemble, $T$ is the temperature, and $V_{\alpha \beta \gamma \delta}\left(\boldsymbol{k}, \boldsymbol{k}^{\prime}\right)$ represents the pair potential. We employ the Green's function approach and rewrite (2) with a use of the anomalous MatsubaraGreen's function $F_{\alpha \beta}\left(\boldsymbol{k}, \omega_{n}\right)$

$$
\Delta_{\alpha \beta}(\boldsymbol{k})=\sum_{\boldsymbol{k}^{\prime}, \gamma, \delta} V_{\alpha \beta \gamma \delta}\left(\boldsymbol{k}, \boldsymbol{k}^{\prime}\right) T \sum_{n} e^{-\mathrm{i} \omega_{n} 0^{+}} F_{\gamma \delta}\left(\boldsymbol{k}^{\prime}, \omega_{n}\right)
$$

where $\omega_{n}$ is the fermionic Matsubara frequency and $F_{\alpha \beta}\left(\boldsymbol{k}, \omega_{n}\right)$ is obtained as a solution of the Gor'kov equations [14]. In a discussion of the critical temperature, $T=T_{c}$, that is at the threshold of superconductivity, we use a linearized anomalous Matsubara-Green's function $F_{\alpha \beta}\left(\boldsymbol{k}, \omega_{n}\right)$ which is determined by the linearized Gor'kov equations for the normal $G_{\alpha \beta}\left(\boldsymbol{k}, \omega_{n}\right)$ and anomalous $F_{\alpha \beta}^{\dagger}\left(\boldsymbol{k}, \omega_{n}\right)=\left(F_{\beta \alpha}\left(\boldsymbol{k},-\omega_{n}\right)\right)^{*}$ temperature Green's functions

$$
\begin{array}{r}
\sum_{\nu}\left[\left(i \omega_{n}-\xi_{\boldsymbol{k}}\right) \delta_{\alpha \nu}-\gamma(\boldsymbol{k}) \cdot \boldsymbol{\sigma}_{\alpha \nu}\right] G_{\nu \beta}\left(\boldsymbol{k}, \omega_{n}\right)=\delta_{\alpha \beta} \\
\sum_{\nu}\left[\left(i \omega_{n}+\xi_{\boldsymbol{k}}\right) \delta_{\alpha \nu}-\gamma(\boldsymbol{k}) \cdot \boldsymbol{\sigma}_{\alpha \nu}^{*}\right] F_{\nu \beta}^{\dagger}\left(\boldsymbol{k}, \omega_{n}\right) \\
+\sum_{\nu} \Delta_{\alpha \nu}^{\dagger}(\boldsymbol{k}) G_{\nu \beta}\left(\boldsymbol{k}, \omega_{n}\right)=0
\end{array}
$$

where $\xi_{\boldsymbol{k}}=\varepsilon_{\boldsymbol{k}}-\mu$. A consistency condition of equations (3) and (4) leads to a general form of the superconducting order parameter $\hat{\Delta}(\boldsymbol{k})$ which consists of the singlet $\Delta_{s} e(\boldsymbol{k})$ and triplet $\Delta_{t} \boldsymbol{d}(\boldsymbol{k})$ components

$$
\hat{\Delta}(\boldsymbol{k})=\left(\Delta_{s} e(\boldsymbol{k}) \hat{\sigma}_{0}+\Delta_{t} \boldsymbol{d}(\boldsymbol{k}) \cdot \hat{\boldsymbol{\sigma}}\right)\left(\mathbf{i} \hat{\sigma}_{y}\right)
$$

with $\Delta_{s}$ and $\Delta_{t}$ representing the temperature dependent amplitudes, and $e(\boldsymbol{k}), \boldsymbol{d}(\boldsymbol{k})$ determining the symmetry of the singlet and triplet order parameters. Introducing a normalized vector $\hat{\gamma}(\boldsymbol{k})=\gamma(\boldsymbol{k}) /|\gamma(\boldsymbol{k})|$ we express the solutions of equation (4) as matrices in the spin-space. The normal Green's function

$$
\hat{G}\left(\boldsymbol{k}, \omega_{n}\right)=\frac{1}{2} \sum_{\lambda= \pm} g_{\lambda}\left(\boldsymbol{k}, \omega_{n}\right)\left[\hat{\sigma}_{0}+\lambda \hat{\gamma}(\boldsymbol{k}) \cdot \hat{\boldsymbol{\sigma}}\right]
$$

is determined by the quasiparticle propagators

$$
g_{\lambda}\left(\boldsymbol{k}, \omega_{n}\right)=\left(i \omega_{n}-\xi_{\boldsymbol{k}}^{\lambda}\right)^{-1}
$$

for the spin-split energy bands defined by $\varepsilon_{\boldsymbol{k}}^{\lambda}=\varepsilon_{\boldsymbol{k}}+$ $\lambda|\gamma(\boldsymbol{k})|$, where $\lambda= \pm$ is the index of the band and $\xi_{\boldsymbol{k}}^{\lambda}=\left(\varepsilon_{\boldsymbol{k}}^{\lambda}-\mu\right)$ represents a corresponding quasiparticle excitation energy. The anomalous Green's function

$$
\hat{\boldsymbol{F}}\left(\boldsymbol{k}, \omega_{n}\right)=\left[\hat{F}_{s}\left(\boldsymbol{k}, \omega_{n}\right)+\left(\hat{\boldsymbol{F}}_{t}\left(\boldsymbol{k}, \omega_{n}\right)+\hat{\boldsymbol{F}}_{\omega_{n}}\left(\boldsymbol{k}, \omega_{n}\right)\right) \cdot \hat{\boldsymbol{\sigma}}\right] \mathrm{i} \hat{\sigma}_{y}
$$

consists of the singlet

$$
\hat{F}_{s}\left(\boldsymbol{k}, \omega_{n}\right)=\frac{1}{2} \sum_{\lambda= \pm} f_{\lambda}\left(\boldsymbol{k}, \omega_{n}\right)\left(\Delta_{s} e(\boldsymbol{k})+\lambda \Delta_{t} \boldsymbol{d}(\boldsymbol{k}) \cdot \hat{\gamma}(\boldsymbol{k})\right)
$$


and triplet

$$
\begin{aligned}
\hat{\boldsymbol{F}}_{t}\left(\boldsymbol{k}, \omega_{n}\right)= & \frac{1}{2} \sum_{\lambda= \pm} f_{\lambda}\left(\boldsymbol{k}, \omega_{n}\right)\left(\frac{\xi_{\boldsymbol{k}}^{\lambda}}{\xi_{\boldsymbol{k}}} \Delta_{t} \boldsymbol{d}(\boldsymbol{k})\right. \\
& \left.-\lambda\left[\Delta_{s} e(\boldsymbol{k})+\frac{\Delta_{t}}{\xi_{\boldsymbol{k}}} \boldsymbol{d}(\boldsymbol{k}) \cdot \gamma(\boldsymbol{k})\right] \hat{\gamma}(\boldsymbol{k})\right)
\end{aligned}
$$

components, where $f_{\lambda}\left(\boldsymbol{k}, \omega_{n}\right)=\left(\omega_{n}^{2}+\left(\xi_{\boldsymbol{k}}^{\lambda}\right)^{2}\right)^{-1}$. The third term of $\hat{\boldsymbol{F}}\left(\boldsymbol{k}, \omega_{n}\right)$

$$
\hat{\boldsymbol{F}}_{\omega_{n}}\left(\boldsymbol{k}, \omega_{n}\right)=\frac{\omega_{n}}{2 \xi_{\boldsymbol{k}}} \Delta_{t} \boldsymbol{d}(\boldsymbol{k}) \times \hat{\gamma}(\boldsymbol{k}) \sum_{\lambda= \pm} \lambda f_{\lambda}\left(\boldsymbol{k}, \omega_{n}\right)
$$

is an odd in $\omega_{n}$ off-diagonal Green's function which supports a creation of the interband Cooper pairs defined by $\boldsymbol{d}(\boldsymbol{k})$ orthogonal to $\gamma(\boldsymbol{k})$. Note that $\hat{\boldsymbol{F}}_{\omega_{n}}\left(\boldsymbol{k}, \omega_{n}\right)$ does not contribute to the gap equation (3) for the frequency independent pairing interaction. We assume that in the scattering of the Cooper pairs determined in equation (3) by $V_{\alpha \beta \gamma \delta}\left(\boldsymbol{k}, \boldsymbol{k}^{\prime}\right)$ the spins are not affected and the pairing potential consists of the singlet $(\mathrm{s})$ and triplet $(\mathrm{t})$ channels

$$
V_{\alpha \beta \gamma \delta}\left(\boldsymbol{k}, \boldsymbol{k}^{\prime}\right)=V_{s_{\alpha \beta \gamma \delta}}\left(\boldsymbol{k}, \boldsymbol{k}^{\prime}\right)+V_{t_{\alpha \beta \gamma \delta}}\left(\boldsymbol{k}, \boldsymbol{k}^{\prime}\right)
$$

which have the following form

$$
V_{i_{\alpha \beta \gamma \delta}}\left(\boldsymbol{k}, \boldsymbol{k}^{\prime}\right)=-\frac{1}{2} V_{i}\left(\boldsymbol{k}, \boldsymbol{k}^{\prime}\right)\left(\delta_{\alpha \delta} \delta_{\beta \gamma} \pm \delta_{\alpha \gamma} \delta_{\beta \delta}\right)
$$

where the,+- -sign occurs for the potential supporting odd $(i=t)$, even $(i=s)$ parity pairing, respectively. Accordingly, we neglect a possible mixed singlet-triplet pairing channel [15] in the pair potential (12) and consider the intrinsic mixed parity coupling stemming directly from the lack of the inversion symmetry. The above assumption does not affect a discussion of the separate singlet and triplet state instabilities induced by the presence of either singlet or triplet pairing in (12). For the pair potential (12) the linearized gap equation (3) can be transformed into a uniform set of $T_{c}$ equations

$$
\begin{aligned}
\Delta_{s} e(\boldsymbol{k})= & \sum_{\lambda= \pm} \sum_{\boldsymbol{k}^{\prime}} V_{s}\left(\boldsymbol{k}, \boldsymbol{k}^{\prime}\right) \frac{1}{4 \xi_{\boldsymbol{k}^{\prime}}^{\lambda}} \tanh \left(\frac{\xi_{\boldsymbol{k}^{\prime}}^{\lambda}}{2 T_{c}}\right) \\
& \times\left(\Delta_{s} e\left(\boldsymbol{k}^{\prime}\right)+\lambda \Delta_{t} \boldsymbol{d}\left(\boldsymbol{k}^{\prime}\right) \cdot \hat{\gamma}\left(\boldsymbol{k}^{\prime}\right)\right) \\
\Delta_{t} \boldsymbol{d}(\boldsymbol{k})= & \sum_{\lambda= \pm} \sum_{\boldsymbol{k}^{\prime}} V_{t}\left(\boldsymbol{k}, \boldsymbol{k}^{\prime}\right) \frac{1}{4 \xi_{\boldsymbol{k}^{\prime}}^{\lambda}} \tanh \left(\frac{\xi_{\boldsymbol{k}^{\prime}}^{\lambda}}{2 T_{c}}\right) \\
& \times\left(\frac{\xi_{\boldsymbol{k}^{\prime}}^{\lambda}}{\xi_{\boldsymbol{k}^{\prime}}} \Delta_{t} \boldsymbol{d}\left(\boldsymbol{k}^{\prime}\right)-\lambda\left[\Delta_{s} e\left(\boldsymbol{k}^{\prime}\right)+\frac{\Delta_{t}}{\xi_{\boldsymbol{k}^{\prime}}} \boldsymbol{d}\left(\boldsymbol{k}^{\prime}\right) \cdot \gamma\left(\boldsymbol{k}^{\prime}\right)\right]\right. \\
& \left.\times \hat{\gamma}\left(\boldsymbol{k}^{\prime}\right)\right)
\end{aligned}
$$

which are accomplished by the relation that determines the chemical potential for a given filling $n$ of the total number of states $2 N_{0}$

$$
n=1-\frac{1}{2 N_{0}} \sum_{\lambda= \pm} \sum_{k} \tanh \left(\frac{\xi_{\boldsymbol{k}}^{\lambda}}{2 T_{c}}\right) .
$$

The set of equations (14)-(16) allows for a fully consistent discussion of the $T_{c}$ dependence on the antisymmetric spin-orbit coupling which encompasses two effects related to the lifted spin degeneracy - the pair-breaking effect and the induced evolution of the spin-split energy band. Since for a finite band width the momentum summations in equations (14) and (15) are convergent without a common for the weak-coupling cut-off procedure, we do not limit the action of the pair potential to the vicinity of the Fermi surface and extend its range to the entire Brillouin zone. This approach does not impose any constraint on the energy of the interacting quasiparticles and accounts for the intraband as well as the interband pairing of the opposite momenta quasiparticles in a two-band system. Note that the creation of the interband Cooper pairs is intensified by the pairing process which involves the Brillouin zone points distant from the Fermi surface and becomes considerable for the amplitude of the pair potential exceeding the magnitude of the hopping integral, that is when superconductivity evolves from the weak-coupling to the intermediate-coupling limit. The $T_{c}$ equations (14) and (15) are solved for the symmetry allowed factorizable pair potentials

$$
V_{s}\left(\boldsymbol{k}, \boldsymbol{k}^{\prime}\right)=V_{s} e(\boldsymbol{k}) e\left(\boldsymbol{k}^{\prime}\right), V_{t}\left(\boldsymbol{k}, \boldsymbol{k}^{\prime}\right)=3 V_{t} \boldsymbol{d}(\boldsymbol{k}) \cdot \boldsymbol{d}\left(\boldsymbol{k}^{\prime}\right)
$$

where the positive pair potential amplitudes $V_{s}$ and $V_{t}$ are assumed to be unaltered by the spin-orbit coupling. This assumption is to be considered a model conjecture, which enables a weak-coupling study of the direct effect of the spin-split single-particle energy spectrum on the critical temperature. Nevertheless, we remark that within the scenario of the strong-coupling Kohn-Luttinger pairing mechanism the increasing spin-orbit coupling rate does not affect the singlet pairing channel and diminishes the amplitude of the pairing interaction for the triplet channel [16]. We employ the presented method to the lowest angular momentum states, i.e., the $s$-wave singlet state $(L=0)$ induced by a momentum independent $V_{s}\left(\boldsymbol{k}, \boldsymbol{k}^{\prime}\right)=$ $V_{s}>0$ and the $p$-wave states, developed by (17) for $\boldsymbol{d}(\boldsymbol{k})$ corresponding to the $L=1$ eigenfuctions. We discuss the uncoupled singlet and triplet state instabilities as well as the onset of the mixed singlet-triplet state. Before starting a thorough analysis, we indicate that in the case of a purely singlet superconductivity $\left(V_{t}\left(\boldsymbol{k}, \boldsymbol{k}^{\prime}\right)=0\right)$ the spinorbit coupling effect on $T_{c}$ (14) is exerted solely through the spin-split quasiparticle energy spectrum, $\xi_{\boldsymbol{k}}^{\lambda}$, and in the weak-coupling approach to superconductivity is exemplified by the induced change of the Fermi surface density of states, which is negligible for the Fermi gas. Accordingly, the Fermi gas approximation applied in references $[6,7]$ leads to a conclusion of the antisymmetric spin-orbit coupling independent critical temperature of a singlet weakcoupling superconductor.

\section{Results}

The computations are performed for a square lattice nearest neighbor tight-binding system defined by a disper$\operatorname{sion} \varepsilon_{\boldsymbol{k}}=-2 t\left(\cos \left(k_{x}\right)+\cos \left(k_{y}\right)\right)$ and for the lowest order 

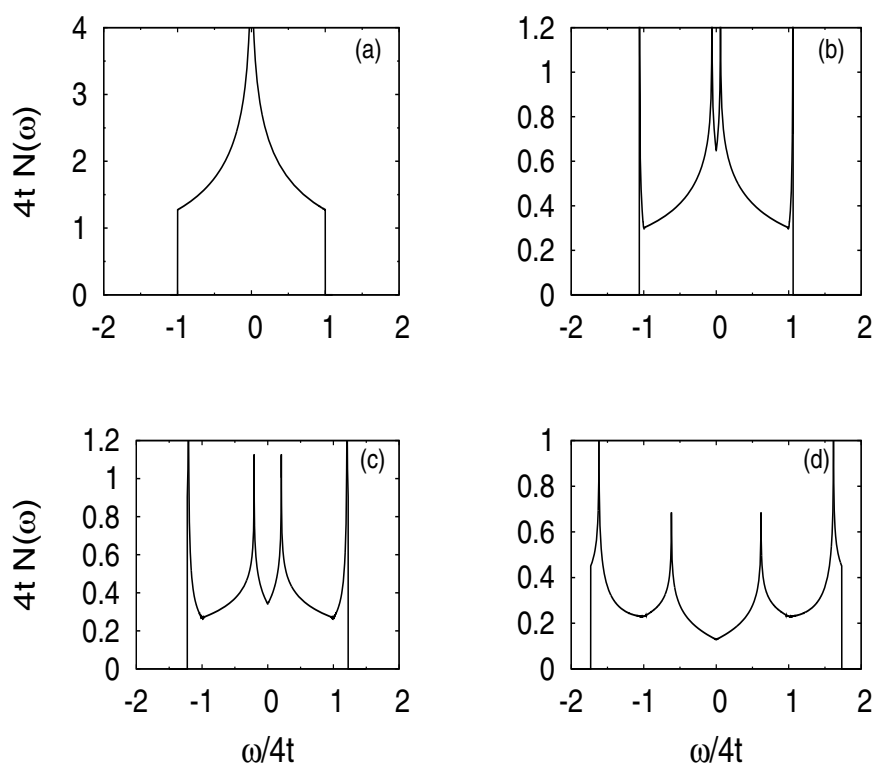

Fig. 1. Dimensionless density of states (normalized to 2) of the nearest neighbor tight-binding square lattice for the spin-orbit coupling rate $\gamma_{0} / t$ : (a) 0 , (b) 1 , (c) 2 , (d) 4 .

Rashba-type spin-orbit coupling represented by $\gamma(\boldsymbol{k})=$ $\gamma_{0}\left(-\hat{\boldsymbol{x}} \sin \left(k_{y}\right)+\hat{\boldsymbol{y}} \sin \left(k_{x}\right)\right)$. This simple model simulates the enhanced width of the spin-split energy band and a concomitant modification of the spectral weights in the density of states including a redistribution of the van Hove singularities. Considering a wide range of magnitude of the model parameters - the band filling $n$ (16), the spin-orbit coupling rate $\gamma_{0}$ and the amplitudes $V_{s}, V_{t}$ of the singlet and triplet pair potentials (17) - we present a systematic study of the antisymmetric spin-orbit coupling effect on the superconducting phase transition. In this respect our approach differs from a comprehensive analysis of the threshold of superconductivity realized in the presence of the Rashba-type spin-orbit interaction at the interface of $\mathrm{Sr}_{2} \mathrm{RuO}_{4}$ [16] which is limited to the appropriate for the considered compound single set of values representing the band filling and the amplitude of the strong coupling pairing potential.

\subsection{Band evolution}

The spin-orbit coupling induced evolution of the energy band is best illustrated by the development of the spinsplit density of states defined as

$$
N(\omega)=\frac{1}{(2 \pi)^{2}} \sum_{\lambda= \pm} \int_{\varepsilon_{\boldsymbol{k}}^{\lambda}=\omega} \frac{d S_{\boldsymbol{k}}}{\left|\nabla \varepsilon_{\boldsymbol{k}}^{\lambda}\right|}
$$

where the integral extends over a constant energy contour in the reciprocal space. We show the antisymmetric spinorbit coupling induced evolution of the density of states of a square lattice system in Figure 1. The singular Brillouin zone points of the density of states are identified by the

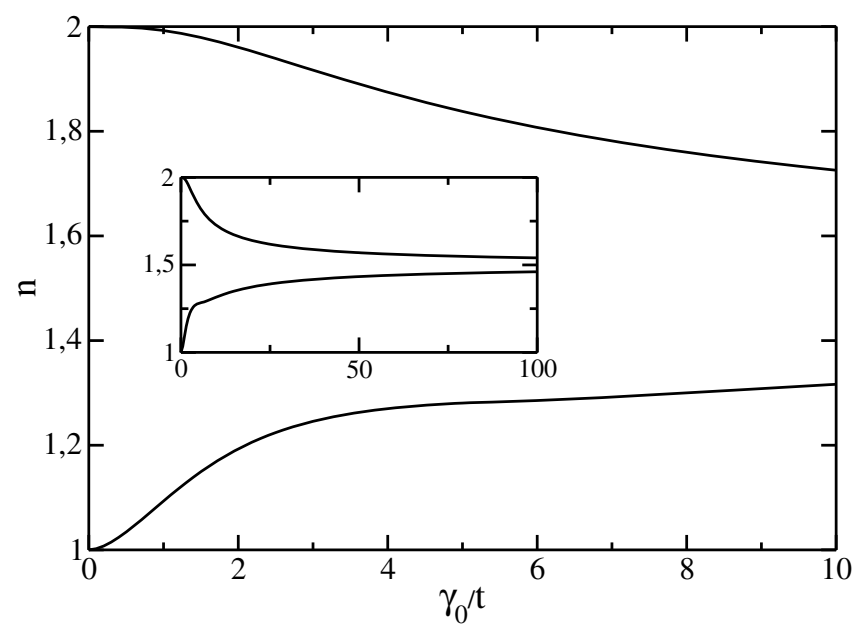

Fig. 2. Band fillings corresponding at the temperature $T / t=10^{-3}$ to the Fermi surface singularities of the noncentrosymmetric nearest neighbor tight-binding square lattice vs. the antisymmetric spin-orbit coupling rate $\gamma_{0}$. The lower curve represents the $\omega_{1}$ singularity and the upper one corresponds to the edge singularity $\omega_{2}$. The inset shows the asymptotic behavior. The $n=2$ filling for $\gamma_{0}=0$ is not singular.

nodes of the velocities, $\nabla \varepsilon_{\boldsymbol{k}=\boldsymbol{k}_{\lambda}}^{\lambda}=0$, and are classified in two groups of points:

(1) $\left(0, k_{\lambda}\right)$ and $\left(k_{\lambda}, 0\right)$, which evolve from the centrosymmetric system singular points $(0, \pm \pi)$ and $( \pm \pi, 0)$ and give rise to singularities at the energy levels

$$
\omega_{1}=\lambda\left(-2 t+\sqrt{4 t^{2}+\gamma_{0}^{2}}\right)
$$

(2) $\left( \pm \pi, k_{\lambda}\right)$ and $\left(k_{\lambda}, \pm \pi\right)$, which emerge from the non singular points of the centrosymmetric system $( \pm \pi, \pm \pi)$ and develop singularities at the edge of the band

$$
\omega_{2}=\lambda\left(2 t+\sqrt{4 t^{2}+\gamma_{0}^{2}}\right) .
$$

Accordingly, the antisymmetric spin-orbit coupling splits the single van Hove singularity of the tight-binding system symmetrically into the particle and hole singularities $\omega_{1}$, resulting in a continuous depletion of the number of states at the band center, and leads to a concomitant gradual enhancement of the spectral weights at the edge of the band where the edge singularities $\omega_{2}$ are developed. The Fermi surface singularities of the density of states arise for $\omega_{1}$ or $\omega_{2}$ coinciding with the chemical potential. We determine the band fillings, $n$, and corresponding antisymmetric spin-orbit coupling rates, $\gamma_{0}$, which lead to the Fermi level singularities $\omega_{1}$ and $\omega_{2}$ from equation (16). This weakly temperature dependent relation is presented in Figure 2 for the particle fillings $(n \geq 1)$. Due to the particle-hole symmetry it can be extended to the hole fillings $(0 \leq n<1)$ by the mirror reflection with respect to the $n=1$ axis. We note that in the limit of the strong spin-orbit coupling the singular band fillings approach asymptotically $n=0.5$ and $n=1.5$ 
which, as well as $n=0$ and $n=2$, do not lead to the van Hove singularities at the Fermi level for the nearest neighbor tight-binding dispersion. Considered in Figure 2 values of the spin-orbit coupling rate $\gamma_{0}$ are used to illustrate a property of the non-centrosymmetric nearest neighbor tight-binding model and should not be identified with the spin-orbit coupling strength in the actual non-centrosymmetric compounds. We note that, the experimentally established ratio of the spin-orbit coupling energy $E_{S O}$ and the Fermi energy $E_{F}$ in the up to date classified non-centrosymmetric compounds does not exceed 0.2. The representative magnitudes of the $E_{S O} / E_{F}$ ratio are 0.017 for $\operatorname{Ir}_{2} \mathrm{Ga} 9$ [17,18], 0.03 for $\mathrm{Li}_{2} \mathrm{Pt}_{3} \mathrm{~B}$ [19] or as high as 0.15 for the heavy fermion compounds $\mathrm{CePt}_{3} \mathrm{Si}, \mathrm{CeRhSi}_{3}$ and $\mathrm{CeIrSi}_{3}$ [20]. A simulation of the typical value of $E_{S O} / E_{F} \sim 0.1$ in our model would require $\gamma_{0} / t \sim 0.2$ for the half-filled band or $\gamma_{0} / t \sim 0.4$ for the band filling $n=1.9$. We emphasize, however, that we do not attempt to give a quantitative analysis of superconducting properties of a specific non-centrosymmetric compound but employ the simplest band model to study the general features of a superconducting phase transition in the presence of a broken inversion symmetry. We indicate that the local changes of the density of states exemplified by the van Hove singularities $\omega_{1}$ and $\omega_{2}$ should result in particularly eminent features for the weak-coupling superconductivity, which is predominantly determined by the Fermi surface density of states. As a result, $\omega_{1}$ or $\omega_{2}$ crossing the Fermi level may give rise to an abrupt enhancement of the weak-coupling critical temperature. On the other hand, a similar $T_{c}$ effect may occur in the intermediate-coupling regime for the Fermi surface singularities which globally dominate the spectral function. The expected $T_{c}$ enhancement should become particularly strong for the $s$-wave singlet state for which, due to the momentum independent pair potential, the Fermi surface momenta contributions add up with a constant sign in the $T_{c}$ equation (14).

\subsection{Singlet s-wave state}

We solve the decoupled $T_{c}$ equation (14) of a singlet state $\left(\Delta_{s} \neq 0, \Delta_{t}=0\right)$

$$
1=\sum_{\lambda= \pm} \sum_{\boldsymbol{k}^{\prime}} V_{s} \frac{e^{2}\left(\boldsymbol{k}^{\prime}\right)}{4 \xi_{\boldsymbol{k}^{\prime}}^{\lambda}} \tanh \left(\frac{\xi_{\boldsymbol{k}^{\prime}}^{\lambda}}{2 T_{c}}\right)
$$

for the momentum-independent $s$-wave state given by the order parameter $e(\boldsymbol{k})=1$ and show the results for the weak- and intermediate-coupling superconductivity in Figure 3 . We use the critical temperature $T_{c_{0}}$ of a system with no spin-orbit coupling to normalize the critical temperature $T_{c}$ and for definiteness, we consider the band filling $n=1.2$ for which the spin-orbit coupling driven singularity $\omega_{1}$ crosses the Fermi energy for the coupling rate $\gamma_{0} / t=2.1$ (Fig. 2). The obtained solutions reveal two types of the critical temperature evolution with the increasing antisymmetric spin-orbit coupling rate which are represented by a monotonic suppression related to the
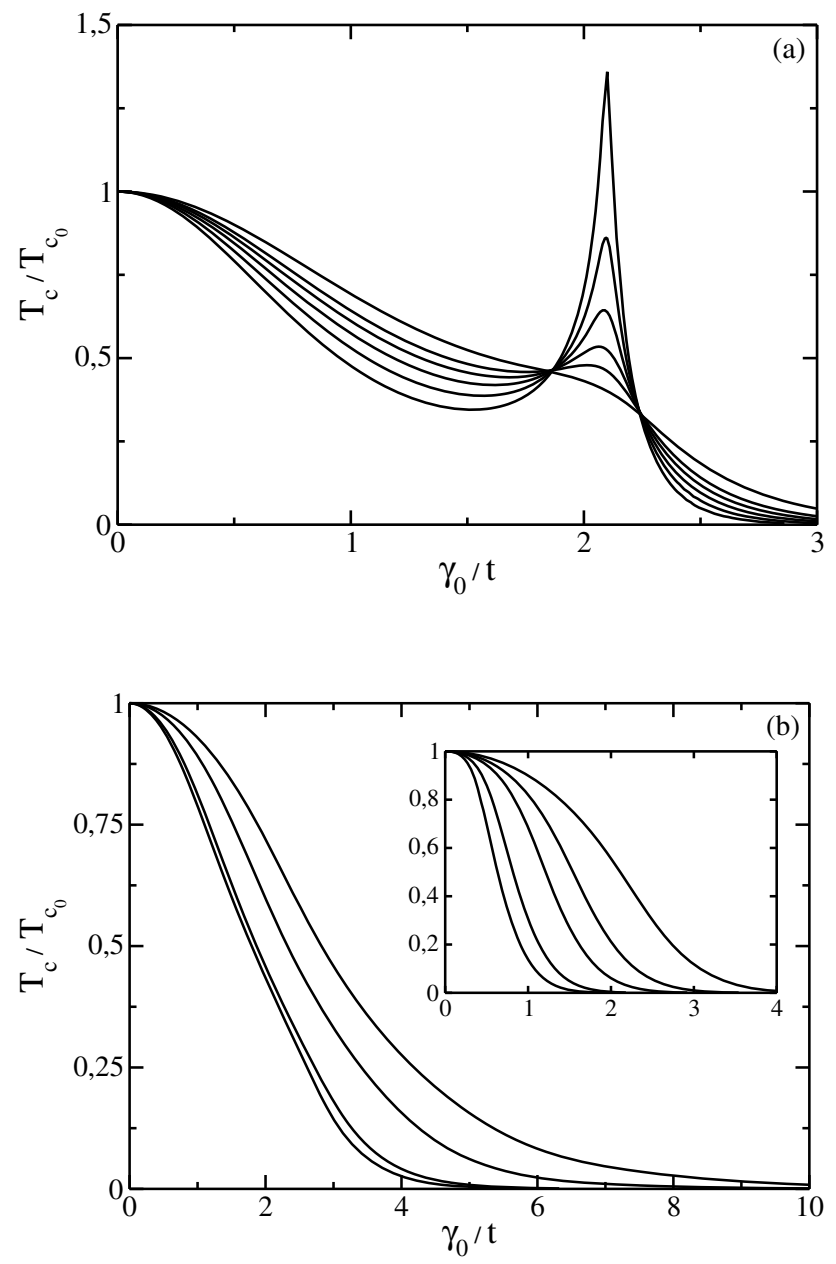

Fig. 3. Critical temperature $T_{c}$ of the $s$-wave state normalized by the critical temperature $T_{c_{0}}$ of a system with no spin-orbit coupling for the band filling $n=1.2$ : (a) weakcoupling pair potential, maxima from top to bottom correspond to $V_{s} / t=0.6,0.7,0.8,0.9,1,1.2$; (b) intermediatecoupling pair potential, curves from top to bottom correspond to $V_{s} / t=3,2,1.8,1.5$. The inset shows $T_{c} / T_{c_{0}}$ of a half-filled system, curves from top to bottom are drawn for the pair potentials $V_{s} / t=3,2,1.5,1,0.8$. Note that $T_{c_{0}}$ depends on $V_{s}$ and is different for each curve.

broadening of the energy band and a peak behavior corresponding to a development of the van Hove singularity at the Fermi level. A comparison of the $T_{c}$ dependence on the spin-orbit coupling rate for various magnitudes of the pair potential $V_{s}$ (Fig. 3) shows that the monotonic reduction as well as the abrupt rise of $T_{c} / T_{c_{0}}$ are intensified by the decreasing pairing strength. In particular, we read from Figure $3 \mathrm{a}$ that, the $T_{c} / T_{c_{0}}$ peak effect is weak for the weak-coupling pair potentials which are relatively strong $\left(V_{s} / t \geq 1\right)$ and involve in the pairing process the single particle states from a large part of the energy band. Such an interaction leads to a formation of a superconducting state relatively robust to the modifications of the Fermi surface density of states. The induced state features then a predominant monotonic suppression of the critical temperature due to the spin-orbit coupling which is caused 
by a thorough depletion of the density of states for a wide energy range. On the other hand, the influence of a local massive shift of the single particle states on the critical temperature is highly pronounced for the weak pairing interactions defined by $V_{s} / t<1$ which give rise to a superconducting state formed predominantly in a close vicinity of the Fermi surface. Accordingly, a gradual decrease of the pair potential strength results in a growing relative contribution of the Fermi surface states in a development of the superconducting state and is manifested by the increasing value of the $T_{c} / T_{c_{0}}$ peak corresponding to the Fermi level singularity of the density of states. The pair potential dependence of the overall suppression as well as of the abrupt rise of $T_{c} / T_{c_{0}}$ leads for the weakcoupling superconductivity to the intersection of all the $T_{c} / T_{c_{0}}$ curves in two pair potential independent points (Fig. 3a). These two universal points are determined by the spin-orbit coupling rates for which the relative critical temperature $T_{c} / T_{c_{0}}$ does not depend on the effective reach of the weak-coupling pair potential in the Brillouin zone. In other words, the densities of states corresponding to the discerned two rates of the antisymmetric spin-orbit coupling maintain a balance between the pairing processes in the direct vicinity of the Fermi surface and in the regions of the Brillouin zone distant from the Fermi level. The overall monotonic suppression of the critical temperature becomes well established for the pair potentials significantly stronger than $V_{s} / t \sim 1$, when superconductivity enters the intermediate-coupling regime and $T_{c}$ is equally determined by the states from the entire Brillouin zone (Fig. 3b).

This type of the $T_{c}$ dependence is also displayed by the weak- and intermediate-coupling superconductors for the band fillings which rule out the Fermi level singularities of the density of states, that is, for $n=0.5,1,1.5$ in the tight-binding model (Fig. 2) as presented for a wide range of the pair potential strength in the inset figure in Figure $3 b$, or when the Fermi surface singularities arise for the strength of the spin-orbit coupling exceeding the critical value which leads to a complete suppression of superconductivity. We also note that, the spin-orbit coupling induced rise of $T_{c}$ is not limited to the weak-coupling superconductivity solely, but may be also featured by the intermediate-coupling superconductors if the spectral weight of the van Hove singularity at the Fermi level dominates the density of states. This condition is fulfilled by the edge singularity $\omega_{2}$ for the band fillings $n \approx 0.1$ or $n \approx 1.9$ (Fig. 2 ) and the strong spin-orbit coupling, $\gamma_{0} / t \approx 3$ (Fig. 1). The critical temperature features then a broad maximum (Fig. 4) instead of a sharp peak. We point out that, the presented in Figures $3 \mathrm{a}$ and $4 \mathrm{~b}$ properties of the $T_{c} / T_{c_{0}}$ diagrams, which include the reduction of the $T_{c} / T_{c_{0}}$ peak value with the increasing strength of the pair potential and the following appearance of two universal points, are characteristic only to the relative change of the critical temperature, that is, $T_{c}$ plotted in the units of the pair potential dependent $T_{c_{0}}$. When measured in the absolute units of the hopping energy, $t$, the superconducting instability temperature remains a monotoni-
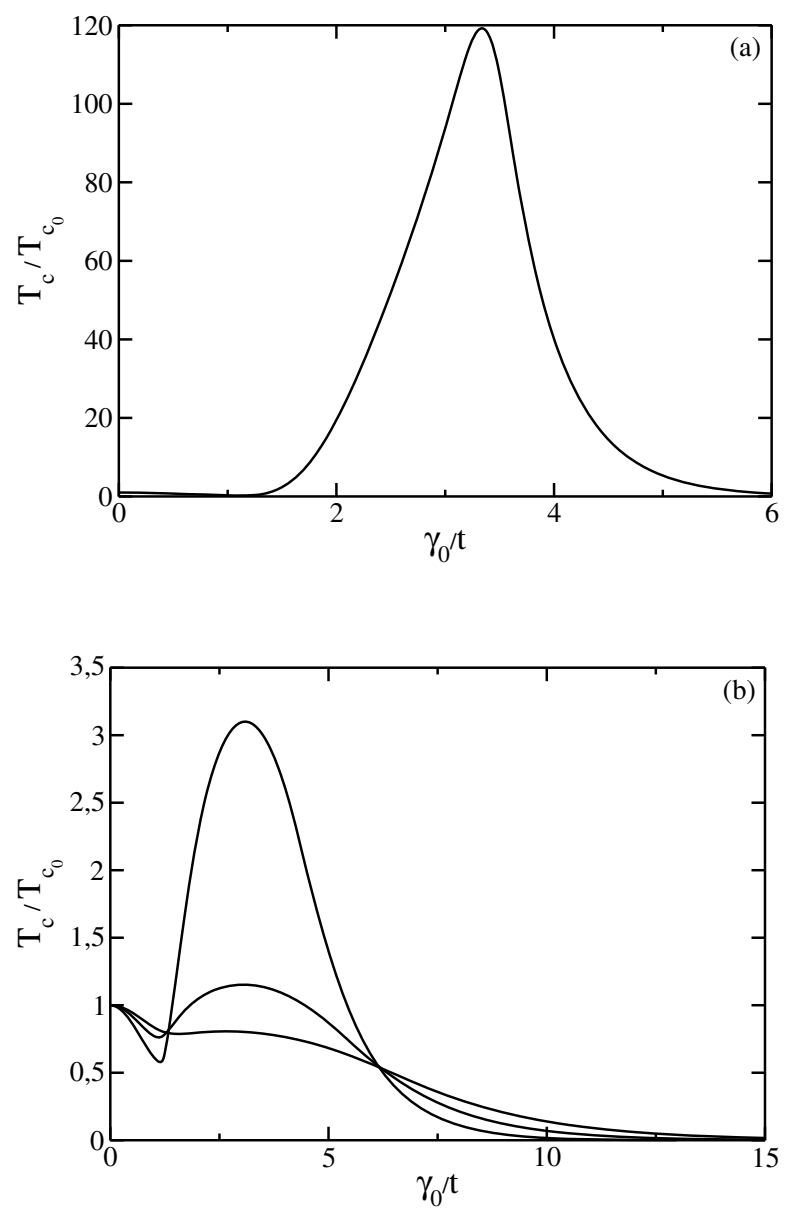

Fig. 4. Critical temperature of an $s$-wave superconductor normalized by the critical temperature $T_{c_{0}}$ of a system with no spin-orbit coupling for the band filling $n=1.9$ : (a) weakcoupling regime $V_{s} / t=1$, (b) intermediate-coupling regime, maxima from top to bottom correspond to $V_{s} / t=2,3,4$.

cally increasing function of the pairing strength for any spin-orbit coupling rate. The absolute $T_{c} / t$ peak values range then in Figure 3a: from $4.7 \times 10^{-4}$ for $V_{s} / t=0.6$ to $6.2 \times 10^{-3}$ for $V_{s} / t=1$, and in Figure 4: from $4.8 \times 10^{-3}$ for the weak-coupling potential $V_{s} / t=1$ to $1.9 \times 10^{-1}$ for the intermediate-coupling potential $V_{s} / t=4$. Concluding we indicate that, our quantitative analysis of the tightbinding $s$-wave state shows the approximately spin-orbit coupling independent $T_{c}$, in agreement with the result of Frigeri et al. [6], for the coupling rate, $\gamma_{0} / t$, which results in a negligible broadening of the energy band and varies in magnitude, depending on the band filling as well as the strength of the pair potential, from $10^{-3}$ for the weak-coupling to $10^{-2}$ for the intermediate-coupling superconductivity. We have discussed the superconducting instability neglecting the orderings induced by the broken translational symmetry - the charge density wave (CDW) and spin density wave (SDW) phases. The onset of these ordered states is driven by the nesting property of the system and depending on the interaction may become dominant for the employed centrosymmetric band structure at half filling due to its perfect nesting property. 
We point out that, the most pronounced effects related to the Fermi surface van Hove singularities which are displayed in Figures $3 \mathrm{a}$ and $4 \mathrm{a}$ were obtained for the band fillings 1.2 and 1.9 to avoid or minimize the effect of competing superconducting and CDW-SDW phases [21,22]. Therefore, we think that this competition is not an issue for these fillings for the pairing potentials adopted in our paper. Furthermore, we note that the increase of the antisymmetric spin-orbit coupling is detrimental to the CDW-SDW phases due to a removal of the nesting condition which stabilizes these phases. In summary, we conclude that the qualitative effect of the peaked critical temperature may be regarded a representative feature of the non-centrosymmetric systems.

\subsection{Triplet $\mathbf{p}$-wave state}

The critical temperature of a triplet superconducting state $\left(\Delta_{s}=0, \Delta_{t} \neq 0\right)$ defined by the order parameter $\boldsymbol{d}(\boldsymbol{k})$ is determined by the decoupled equation (15)

$$
\begin{aligned}
\boldsymbol{d}(\boldsymbol{k})= & \sum_{\lambda= \pm} \sum_{\boldsymbol{k}^{\prime}} V_{t}\left(\boldsymbol{k}, \boldsymbol{k}^{\prime}\right) \frac{1}{4 \xi_{\boldsymbol{k}^{\prime}}^{\lambda}} \tanh \left(\frac{\xi_{\boldsymbol{k}^{\prime}}^{\lambda}}{2 T_{c}}\right) \\
& \times\left(\frac{\xi_{\boldsymbol{k}^{\prime}}^{\lambda}}{\xi_{\boldsymbol{k}^{\prime}}} \boldsymbol{d}\left(\boldsymbol{k}^{\prime}\right)-\frac{\lambda}{\xi_{\boldsymbol{k}^{\prime}}}\left[\boldsymbol{d}\left(\boldsymbol{k}^{\prime}\right) \cdot \boldsymbol{\gamma}\left(\boldsymbol{k}^{\prime}\right)\right] \hat{\gamma}\left(\boldsymbol{k}^{\prime}\right)\right) .
\end{aligned}
$$

For the pair potential (17) the degeneracy of the superconducting states is lifted by the coupling term, $\boldsymbol{d}(\boldsymbol{k}) \cdot \boldsymbol{\gamma}(\boldsymbol{k})$, which reveals to what extent the spin texture of the noncentrosymmetric system is destructive to the defined by the $\boldsymbol{d}(\boldsymbol{k})$-vector spin structure of a triplet state. In other words, we may say that this coupling term manifests the antisymmetric spin-orbit coupling induced pair-breaking effect of a lifted spin-degeneracy which forbids a creation of a Cooper pair by two degenerate quasiparticles of equal spin projection with respect to the $\gamma(\boldsymbol{k})$-axis. Such a most suppressed state corresponding to $\boldsymbol{d}(\boldsymbol{k}) \cdot \boldsymbol{\gamma}(\boldsymbol{k})=0$ is developed then by the interband pairing interaction. We discuss this issue in more detail in Section 3.4. On the other hand, the triplet state of spin projection zero with respect to the $\gamma(\boldsymbol{k})$-axis, which is defined by $\boldsymbol{d}(\boldsymbol{k}) \| \boldsymbol{\gamma}(\boldsymbol{k})$, is not directly affected by the lifted spin degeneracy and the only influence of the spin-orbit coupling upon this developed in two spin-split bands state is exerted by the modified spin-split density of states. We indicate that, the $T_{c}$ equation $(22)$ of the $\boldsymbol{d}(\boldsymbol{k}) \| \boldsymbol{\gamma}(\boldsymbol{k})$ triplet state, which is developed, similarly to the singlet one, by the opposite spin projection quasiparticles, reduces to the critical temperature equation of the singlet state (21). Therefore within the weak-coupling approach assuming a constant Fermi surface value of the density of states the $\boldsymbol{d}(\boldsymbol{k}) \| \boldsymbol{\gamma}(\boldsymbol{k})$ state, similarly to the singlet one, is characterized by the spin-orbit coupling independent critical temperature. We recover here the result of Frigeri et al. [6] derived for the Fermi gas system. In general, the spin-orbit coupling induced evolution of the $\boldsymbol{d}(\boldsymbol{k}) \nVdash \boldsymbol{\gamma}(\boldsymbol{k})$ states is determined by the level of alignment of the order parameter and the antisymmetric spin-orbit coupling vector, and results in
Table 1. Triplet $p$-wave states for the $C_{4 v}$ crystals $[6,23,24]$.

\begin{tabular}{ccc}
\hline$C_{4 v}$ & $\boldsymbol{d}(\boldsymbol{k})$ & $\boldsymbol{d}(\boldsymbol{k}) \cdot \boldsymbol{\gamma}(\boldsymbol{k}) / \gamma_{0}$ \\
\hline$A_{1}$ & $\hat{\boldsymbol{x}} \sin \left(k_{x}\right)+\hat{\boldsymbol{y}} \sin \left(k_{y}\right)$ & 0 \\
$A_{2}$ & $-\hat{\boldsymbol{x}} \sin \left(k_{y}\right)+\hat{\boldsymbol{y}} \sin \left(k_{x}\right)$ & $\sin ^{2}\left(k_{x}\right)+\sin ^{2}\left(k_{y}\right)$ \\
$B_{1}$ & $-\hat{\boldsymbol{x}} \sin \left(k_{x}\right)+\hat{\boldsymbol{y}} \sin \left(k_{y}\right)$ & $\sin \left(k_{x}\right) \sin \left(k_{y}\right)$ \\
$B_{2}$ & $\hat{\boldsymbol{x}} \sin \left(k_{y}\right)+\hat{\boldsymbol{y}} \sin \left(k_{x}\right)$ & $\sin ^{2}\left(k_{x}\right)-\sin ^{2}\left(k_{y}\right)$ \\
\hline
\end{tabular}

a suppression highly dependent on the nodal structure of $\boldsymbol{d}(\boldsymbol{k}) \cdot \boldsymbol{\gamma}(\boldsymbol{k})$. We solve equation (22) for the weak- and intermediate-coupling tetragonal $p$-wave states which are listed in Table 1 and may characterize superconductivity in $\mathrm{CePt}_{3} \mathrm{Si}$ and $\mathrm{CeTX}_{3}$ compounds [2]. Note that a quantitative discussion of the $\mathrm{CePt}_{3} \mathrm{Si}$ requires an extension of the applied nearest neighbor band model to the next nearest neighbor one [25]. The $\boldsymbol{d}(\boldsymbol{k}) \cdot \boldsymbol{\gamma}(\boldsymbol{k})$ nodal places for the considered states consist of the point nodes for the $A_{2}$ state, which is defined by $\boldsymbol{d}(\boldsymbol{k})$ aligned with $\gamma(\boldsymbol{k})$, and the line nodes for the $\boldsymbol{d}(\boldsymbol{k}) \nVdash \boldsymbol{\gamma}(\boldsymbol{k})$ states: $B_{1}$ and $B_{2}$. Note that, for the $A_{1}$ state the degeneracy lifting term vanishes identically in the entire Brillouin zone. We indicate that, the orientation of the nodal structure of the $\boldsymbol{d}(\boldsymbol{k}) \cdot \gamma(\boldsymbol{k})$ parameter with respect to the Fermi surface as well as the geometric measure of the nodal places lead to the following general arrangement of the critical temperatures of considered triplet states: $T_{c}\left(A_{1}\right)<T_{c}\left(B_{2}\right)<T_{c}\left(B_{1}\right)<T_{c}\left(A_{2}\right)$ (Figs. 5 and 6).

We start a detailed discussion of the onset of triplet superconductivity with the half-filled band system for which the Fermi level is fixed at the center of the band and the $\omega_{1}$ van Hove singularity (19) is driven away from the Fermi surface by the increasing rate of the spin-orbit coupling. Since the pair potential vanishes at the singular for the centrosymmetric system Fermi surface points $(0, \pm \pi)$ and $( \pm \pi, 0)$ the contribution of the van Hove singularity to the weak-coupling critical temperature is minimized for $\gamma_{0}=0$ and is expected to be enhanced by a moderately increased spin-orbit coupling rate which shifts singularity into the immediate vicinity of the Fermi surface. Such an initial rise of $T_{c}$, generated by a displacement of the density of states singular points from the nodes of the order parameter, is displayed by the most stable weak-coupling $A_{2}$ state (Fig. 5). We note that any pairing interaction odd in the momentum channel by symmetry vanishes at the Brillouin zone points $(0, \pm \pi)$ and $( \pm \pi, 0)$. Therefore the feature of the slight initial $T_{c}$ enhancement is not limited to a particular $p$-wave pairing but is characteristic to any triplet pairing potential.

For the weak-coupling pairing we also observe a strong suppression of the $A_{1}$ and $B_{2}$ states whose critical temperatures follow the same suppression curve as shown in Figure $5 \mathrm{~b}$ for the pair potential $V_{t} / t=1$. These states are not displayed in Figure 5 a for their very low critical $\gamma_{0} / t$ value which attains $6 \times 10^{-4}$ for $V_{t} / t=0.6$. The approximate degeneracy of the weak-coupling $A_{1}$ and $B_{2}$ states follows from the structure of the degeneracy lifting term $\boldsymbol{d}(\boldsymbol{k}) \cdot \boldsymbol{\gamma}(\boldsymbol{k})$ (Tab. 1) which vanishes on the Fermi surface of a half-filled system for both states. Eventually, their critical temperatures split (Fig. 6) for the pair potential 

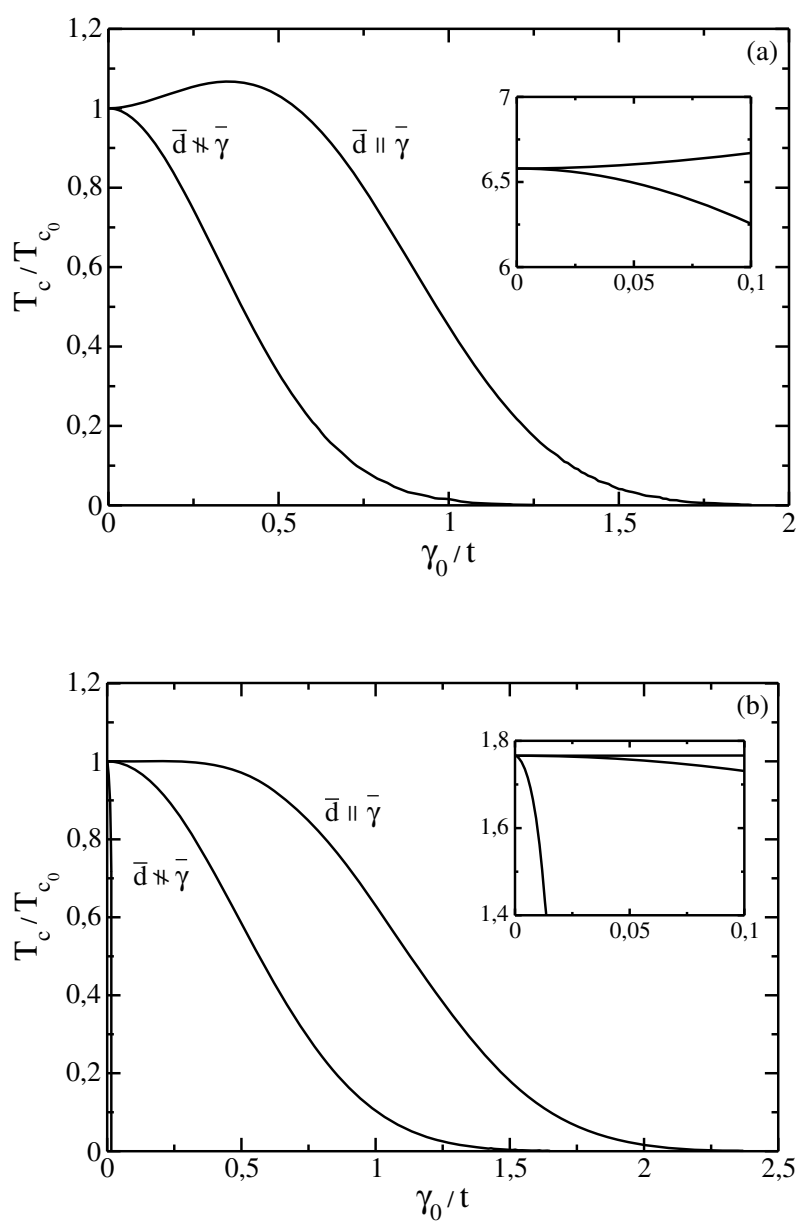

Fig. 5. Critical temperature $T_{c}$ of the two-dimensional $p$-wave states normalized by the critical temperature $T_{c_{0}}$ of a system with no spin-orbit coupling for the half-filled band in the weakcoupling limit: (a) $V_{t} / t=0.6$, (b) $V_{t} / t=1$. The top curve represents the $A_{2}$ state defined by $\boldsymbol{d}(\boldsymbol{k})$ parallel to $\gamma(\boldsymbol{k})$, other curves from top to bottom correspond to the states nonparallel to $\gamma(\boldsymbol{k}): B_{1}$ and $B_{2}$ (not seen in (a)). The $A_{1}$ state is approximately degenerate with the $B_{2}$ state. The insets: (a) $T_{c}$ in the units of $10^{-4} t$ vs. $\gamma_{0} / t$, (b) $T_{c}$ in the units of $10^{-2} t$ vs. $\gamma_{0} / t$.

entering the intermediate-coupling regime $\left(V_{t} / t>1\right)$ when the pairing process is extended over the Brillouin zone section where a non vanishing product $\boldsymbol{d}(\boldsymbol{k}) \cdot \boldsymbol{\gamma}(\boldsymbol{k})$ discerns the $B_{2}$ and $A_{1}$ states. For the intermediate-coupling pairing the initial rise of the critical temperature of the $A_{2}$ state is replaced by the monotonic suppression dependence comparable to that of the $B_{1}$ state (Fig. 6), which as well as other $\boldsymbol{d}(\boldsymbol{k}) \nVdash \boldsymbol{\gamma}(\boldsymbol{k})$ states $-A_{1}$ and $B_{2}$ - is relatively strengthen due to the extended reach of the pair potential in the Brillouin zone. The inset figures of Figures 5 and 6 display the development of the critical temperature given in the units of $t$ for a narrow spin-orbit coupling range, $\gamma_{0} / t \leq 10^{-1}$, corresponding, for discussed critical temperatures, $T_{c_{0}} / t \sim 10^{-4} \div 10^{-2}$, to the reach of the pair-breaking parameter $\gamma_{0} / \pi T_{c_{0}}$ considered in Figure 1 of reference [6]. Comparing our half-filled band solutions with the results of Frigeri et al. presented in Figure 1 of reference [6] we indicate that it is only a solution for the
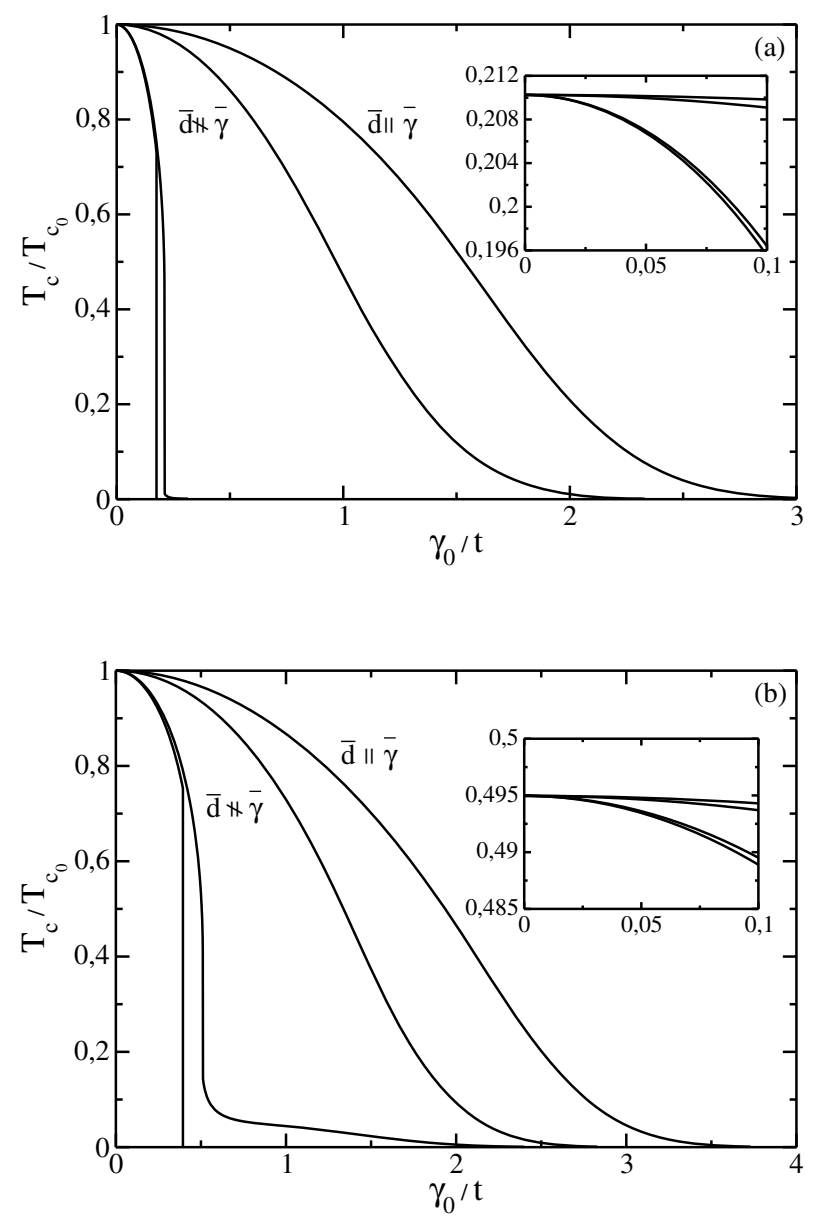

Fig. 6. Critical temperature $T_{c}$ of the two-dimensional $p$ wave states for the half-filled band in the intermediate-coupling limit: (a) $V_{t} / t=2$, (b) $V_{t} / t=3$. The curves from top to bottom correspond to the states: $A_{2}, B_{1}, B_{2}, A_{1}$. The $T_{c}$ in the units of $t$ for low $\gamma_{0}$ is displayed in the insets.

pair potential $V_{t} / t \sim 1$ (Fig. $5 \mathrm{~b}$ ) that resembles the result of reference [6] of an approximately unaltered $T_{c}$ of the $\boldsymbol{d}(\boldsymbol{k}) \| \boldsymbol{\gamma}(\boldsymbol{k})$ state. There are still, however, some discrepancies between this particular case displayed in Figure $5 \mathrm{~b}$ and the result of Frigeri et al. [6] concerning the evolution of the tetragonal periodic states $B_{1}, B_{2}$ (Tab. 1) and their non periodic Fermi gas counterparts defined by $\boldsymbol{d}(\boldsymbol{k})=-\hat{\boldsymbol{x}} k_{x}+\hat{\boldsymbol{y}} k_{y}$ and $\boldsymbol{d}(\boldsymbol{k})=\hat{\boldsymbol{x}} k_{y}+\hat{\boldsymbol{y}} k_{x}$, respectively. Whereas, the suppression of the periodic $B_{1}$ state is comparable to the reduction of the most stable $A_{2}$ state and the $B_{2}$ state becomes approximately degenerate with the most severely suppressed $A_{1}$ state (Fig. 5b), the Fermi gas $B_{1}$ and $B_{2}$ states, displayed in Figure 1 of reference [6], remain degenerate and their suppression is significantly stronger than the reduction of the $A_{2}$ state and considerably weaker than the depletion of the $A_{1}$ state. We note that, the $T_{c}$ suppression curves for the pair potential strengths differing from $V_{t} / t \sim 1$, shown in the inset figures of Figures 5 and 6 , do not coincide with the result of Frigeri et al. [6]. When the band filling departures from $n=1$ we observe a significant suppression of the critical temperature of all states, which is generated 


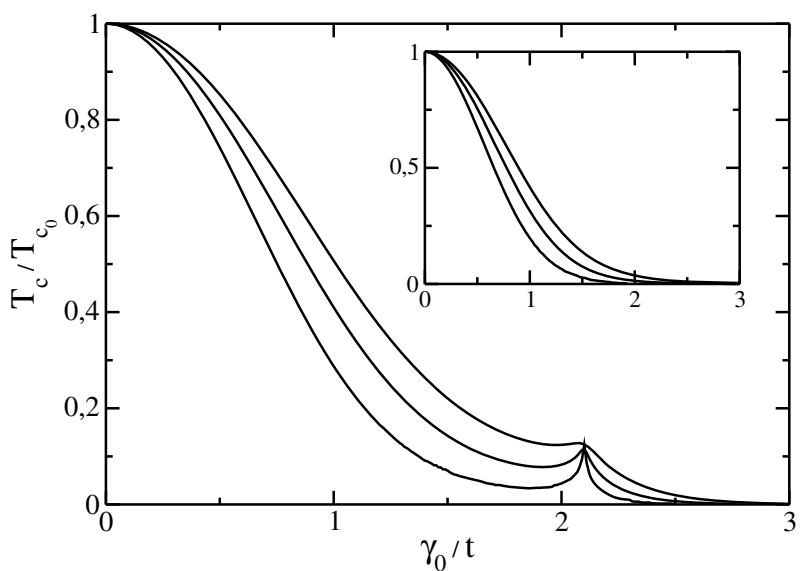

Fig. 7. Weak-coupling critical temperature of the $A_{2}$ state for a singular $(n=1.2)$ and in the inset figure for a non singular $(n=1.3)$ Fermi surface density of states. The curves from from bottom to top correspond to the pair potentials $V_{t} / t=$ $0.6,0.8,1$.

by the broadening of the energy band and a resulting depletion of the density of states in the vicinity of the Fermi surface, unless the density of states features singularities at the Fermi level. In the case of a singular Fermi surface density of states, a sudden rise of the critical temperature for the weak-coupling pair potential is exhibited, however, the feature of the universal pair potential independent points in the phase diagram which are displayed by the $s$-wave superconductivity (Figs. 3a and $4 \mathrm{~b}$ ) is smeared by the presence of the nodes in the pairing interaction. We present the spin-orbit coupling induced development of the $A_{2}$ state for the singular $n=1.2$ and the non singular $n=1.3$ band fillings in Figure 7 and note that, they do not reproduce the result of reference [6]. In summary, we have shown that the triplet states, similarly to the singlet ones, are suppressed by the antisymmetric spin-orbit coupling, however, the nodal structure of the triplet order parameter combined with the band structure may result in some cases in a departure from a monotonic initial $T_{c}$ reduction. We have established that for the square lattice nearest neighbor tight-binding dispersion the behavior of a non decreasing critical temperature for the low spin-orbit coupling rate is featured by the $\boldsymbol{d}(\boldsymbol{k}) \| \boldsymbol{\gamma}(\boldsymbol{k})$ state for the half-filled band and for the weak-coupling pair potential, $V_{t} / t \leq 1$.

\subsection{Intraband vs. interband pairing}

We have considered the pairing interaction of the opposite momenta quasiparticles which leads to the creation of the zero center-of-mass momentum Cooper pairs in a two-band system and in consequence, to a formation of a uniform superconducting state. Such an interaction which does not impose any additional constraint on the energies of the involved quasiparticles may result in the intraband superconductivity for equal energies as well as the interband superconductivity when the Cooper pairs are created by the quasiparticles which differ in energy. The intraband Cooper pair states can be identified as a superposition of the two-particle intraband states $|\boldsymbol{k} \uparrow,-\boldsymbol{k} \downarrow\rangle$ and $|\boldsymbol{k} \downarrow,-\boldsymbol{k} \uparrow\rangle$, each of them developed in a separate spin-split band, which constitute a superconducting state of spin projection zero with respect to the momentum-dependent quantization $\gamma(\boldsymbol{k})$-axis. Accordingly, the intraband pairing leads to a formation of the singlet and $\boldsymbol{d}(\boldsymbol{k}) \| \boldsymbol{\gamma}(\boldsymbol{k})$ triplet states. For the considered $C_{4 v}$ symmetry (Tab. 1) the intraband triplet superconductivity is exemplified by the most stable $A_{2}$ state. In general, the triplet order parameter $\boldsymbol{d}(\boldsymbol{k})=\boldsymbol{d}_{\|}(\boldsymbol{k})+\boldsymbol{d}_{\perp}(\boldsymbol{k})$, where the $\boldsymbol{d}_{\|}(\boldsymbol{k})$ and $\boldsymbol{d}_{\perp}(\boldsymbol{k})$ vectors represent the components parallel and orthogonal to the spin-orbit coupling $\gamma(\boldsymbol{k})$-vector, respectively. The absolute value of the $\boldsymbol{d}_{\|}(\boldsymbol{k})$-vector, which determines the axis with respect to which the triplet Cooper pairs are in a state of spin projection zero, provides a contribution of the intraband counterpart in a mixed intra- and interband state for each point of the Brillouin zone. The triplet superconducting states which are not aligned with $\gamma(\boldsymbol{k})$ and consist of both the intraband and interband Cooper pairs are represented in Figures 5 and 6 by the tetragonal $B_{1}$ and $B_{2}$ states (Tab. 1). We indicate that the interband counterpart, defined by $\boldsymbol{d}(\boldsymbol{k}) \perp \gamma(\boldsymbol{k})$ and developed by the pairing interaction between the quasiparticles of different energies, is strongly suppressed when the pairing process is reduced predominantly to the Fermi surface and becomes more robust for the reach of the pairing interaction extended to the entire energy band. This characteristic feature of the interband state is displayed by the tetragonal $A_{1}$ state (Tab. 1) which reveals a strong suppression in the regime of the weak-coupling superconductivity (Fig. 5) and shows a significantly more moderate reduction of the critical temperature in the regime of the intermediate-coupling superconductivity (Fig. 6). Characteristic for such a state is an abrupt change of the critical temperature from a finite value to zero when the spin-orbit coupling strength $\gamma_{0}$ leads to a band separation energy exceeding the pairing energy and inhibits the interband pairing.

\subsection{Coupled singlet-triplet superconductivity}

In this section we discuss a possible manifestation of the mixed singlet-triplet state defined by the nonzero solutions $\Delta_{s}$ and $\Delta_{t}$ of the coupled set of $T_{c}$ equations (14) and (15). We introduce the same terminology as in [15] and use the term dominant channel to denote the even parity (singlet) and odd parity (triplet) pairing channels responsible for the superconducting instability and call subdominant the spin-orbit coupling induced mixed parity (singlet-triplet) channel. We apply the same convention to the order parameters and use terms dominant and subdominant for the order parameter which initiates the superconducting transition in a centrosymmetric system and the coupled order parameter which is developed due to the antisymmetric spin-orbit coupling, respectively. We start our analysis by indicating that equations (14) and (15) are coupled and determine the critical temperature of a superconducting 
state formed by a superposition of the singlet and triplet states for $\boldsymbol{d}(\boldsymbol{k})$ parallel to $\gamma(\boldsymbol{k})$, whereas for $\boldsymbol{d}(\boldsymbol{k})$ orthogonal to $\gamma(\boldsymbol{k})$ these equations are independent and lead to two different critical temperatures of separate singlet and triplet states. In the most general case of the triplet order parameter consisting of both the parallel, $\boldsymbol{d}_{\|}(\boldsymbol{k})$, and orthogonal, $\boldsymbol{d}_{\perp}(\boldsymbol{k})$, components the set of coupled equations (14) and (15) determines the critical temperature of a superconducting state developed by the superposition of $\Delta_{s} e(\boldsymbol{k})$ and $\Delta_{t} \boldsymbol{d}_{\|}(\boldsymbol{k})$, while the $\boldsymbol{d}_{\perp}(\boldsymbol{k})$ counterpart remains split and its critical temperature is given by the reduced equation (22). Therefore, in order to focus on the singlet-triplet coupling process we discuss the triplet state defined by the $\boldsymbol{d}(\boldsymbol{k})$-vector aligned with the $\gamma(\boldsymbol{k})$-vector. In this case equations (14) and (15) reduce to the set of equations

$$
\begin{aligned}
\Delta_{s} e(\boldsymbol{k})= & \sum_{\lambda= \pm} \sum_{\boldsymbol{k}^{\prime}} V_{s}\left(\boldsymbol{k}, \boldsymbol{k}^{\prime}\right) \frac{1}{4 \xi_{\boldsymbol{k}^{\prime}}^{\lambda}} \tanh \left(\frac{\xi_{\boldsymbol{k}^{\prime}}^{\lambda}}{2 T_{c}}\right) \\
& \times\left(\Delta_{s} e\left(\boldsymbol{k}^{\prime}\right) \pm \lambda \Delta_{t} d\left(\boldsymbol{k}^{\prime}\right)\right) \\
\Delta_{t} \boldsymbol{d}(\boldsymbol{k})= & \sum_{\lambda= \pm} \sum_{\boldsymbol{k}^{\prime}} V_{t}\left(\boldsymbol{k}, \boldsymbol{k}^{\prime}\right) \frac{1}{4 \xi_{\boldsymbol{k}^{\prime}}^{\lambda}} \tanh \left(\frac{\xi_{\boldsymbol{k}^{\prime}}^{\lambda}}{2 T_{c}}\right) \\
& \times\left(\mp \lambda \Delta_{s} e\left(\boldsymbol{k}^{\prime}\right)+\Delta_{t} d\left(\boldsymbol{k}^{\prime}\right)\right) \frac{\boldsymbol{d}\left(\boldsymbol{k}^{\prime}\right)}{d\left(\boldsymbol{k}^{\prime}\right)}
\end{aligned}
$$

where $d(\boldsymbol{k})=|\boldsymbol{d}(\boldsymbol{k})|$ and the upper (lower) sign corresponds to $\boldsymbol{d}(\boldsymbol{k})$ parallel (antiparallel) to $\gamma(\boldsymbol{k})$. Accordingly, the sign of the ratio $\Delta_{t} / \Delta_{s}$ changes to the opposite for the alignment of the $\boldsymbol{d}(\boldsymbol{k})$ and $\gamma(\boldsymbol{k})$ vectors switching from the parallel to the antiparallel. The uniform set of equations (23) and (24) leads to the nonzero $\Delta_{s}, \Delta_{t}$ solutions when its main determinant vanishes. This condition determines the critical temperature of the mixed singlet-triplet state. We write the $T_{c}$ equation for the factorizable pair potential (17) which explicitly displays the symmetry of the ordered state

$$
\begin{gathered}
{\left[1-V_{s} \sum_{\boldsymbol{k}} e^{2}(\boldsymbol{k}) f_{+}\left(\varepsilon_{\boldsymbol{k}}\right)\right]\left[1-V_{t} \sum_{\boldsymbol{k}} d^{2}(\boldsymbol{k}) f_{+}\left(\varepsilon_{\boldsymbol{k}}\right)\right]} \\
=-V_{s} V_{t}\left[\sum_{\boldsymbol{k}} e(\boldsymbol{k}) d(\boldsymbol{k}) f_{-}\left(\varepsilon_{\boldsymbol{k}}\right)\right]^{2}
\end{gathered}
$$

where to shorten the notation we have defined

$$
\begin{aligned}
& f_{+}\left(\varepsilon_{\boldsymbol{k}}\right)=\sum_{\lambda= \pm} \frac{1}{4 \xi_{\boldsymbol{k}}^{\lambda}} \tanh \left(\frac{\xi_{\boldsymbol{k}}^{\lambda}}{2 T_{c}}\right) \\
& f_{-}\left(\varepsilon_{\boldsymbol{k}}\right)=\sum_{\lambda= \pm} \frac{\lambda}{4 \xi_{\boldsymbol{k}}^{\lambda}} \tanh \left(\frac{\xi_{\boldsymbol{k}}^{\lambda}}{2 T_{c}}\right)
\end{aligned}
$$

which are functions of $\varepsilon_{\boldsymbol{k}}, \mu, \gamma$, and $T_{c}$. We note that, the $f_{+}\left(\varepsilon_{\boldsymbol{k}}\right)$ function represents the energy dependence of the singlet and triplet pairings in a two-band system and determines a stability of the singlet $\left(\Delta_{s} \neq 0, \Delta_{t}=0\right)$ and triplet $\left(\Delta_{s}=0, \Delta_{t} \neq 0\right)$ dominant state solutions of equations (23) and (24). On the other hand, the pairing in the mixed singlet-triplet channel which is responsible for the occurrence of the mixed singlet-triplet state $\left(\Delta_{s} \neq 0\right.$, $\left.\Delta_{t} \neq 0\right)$ is exemplified by the $f_{-}\left(\varepsilon_{\boldsymbol{k}}\right)$ function. This function along with the symmetry of the singlet and triplet states determines the magnitude of the non positive coupling term on the right-hand side of equation (25). We indicate that, for a vanishing coupling term the $T_{c}$ equation of the mixed state $(25)$ reduces to the separate $T_{c}$ equations of the uncoupled singlet $(21)$ and $\boldsymbol{d}(\boldsymbol{k}) \| \boldsymbol{\gamma}(\boldsymbol{k})$ triplet (22) states. Accordingly, a nonzero negative value of the term on the right-hand side of equation (25) becomes crucial for the development of the mixed state and constitutes a necessary condition for the coupling of the singlet and triplet states. Based on the properties of the $f_{-}\left(\varepsilon_{\boldsymbol{k}}\right)$ function and using general symmetry arguments we can draw conclusions concerning the existence of the singlet-triplet mixed state. In the following, we conclude that there is no singlet-triplet coupling for:

- a centrosymmetric system, since $\xi_{\boldsymbol{k}}^{\lambda}=\xi_{\boldsymbol{k}}$ and $f_{-}\left(\varepsilon_{\boldsymbol{k}}\right)=0$

- a particle-hole symmetric system for the half-filled conduction band, because $f_{-}\left(\varepsilon_{\boldsymbol{k}}\right)=f_{-}\left(-\varepsilon_{\boldsymbol{k}}\right)$ for $\mu=0$ and the momentum summation on the right-hand side of equation (25) vanishes,

- a singlet state $e(\boldsymbol{k})$ which transforms according to a non-identity representation of the system symmetry point group, for example the $d_{x^{2}-y^{2}}$ state for the $C_{4 v}$ point group, as it is orthogonal to the function $d(\boldsymbol{k}) f_{-}\left(\varepsilon_{\boldsymbol{k}}\right)$ belonging to the identity representation,

- degenerate singlet and triplet states, for which the lefthand side of equation (25) cannot be negative.

We conclude that, the symmetry arguments limit the singlet-triplet coupling to the singlet states belonging to the $A_{1}$ representation. The only constraint on the triplet state is that the $\boldsymbol{d}(\boldsymbol{k})$-vector cannot be orthogonal to the spin-orbit coupling vector $\gamma(\boldsymbol{k})$. However, the highest critical temperature of the mixed state emerging from the dominant triplet state will correspond to $\boldsymbol{d}(\boldsymbol{k})$ aligned with $\gamma(\boldsymbol{k})$. On the other hand, the same relative orientation of the $\boldsymbol{d}(\boldsymbol{k})$ and $\gamma(\boldsymbol{k})$ vectors will lead to the lowest critical temperature of the mixed singlet-triplet state which stems from the dominant singlet state. The appearance of two critical temperatures $T_{c}\left(\Delta_{s}, \Delta_{t}\right)_{s}$ and $T_{c}\left(\Delta_{s}, \Delta_{t}\right)_{t}$ which identify the phase transitions to the mixed singlettriplet states emerging from the pure singlet and pure triplet state, respectively, is a characteristic feature of the antisymmetric spin-orbit coupling (Figs. 8 and 9). These critical temperatures merge at the edge value of the spinorbit coupling which leads to the maximal relative magnitude of the subdominant order parameter for a given dominant pairing strength. The pairing energy provided by the pair potentials $V_{s}$ and $V_{t}$ is saturated then by the largest subdominant components which become equivalent to the dominant ones. We indicate that, both critical temperatures $T_{c}\left(\Delta_{s}, \Delta_{t}\right)_{s}$ and $T_{c}\left(\Delta_{s}, \Delta_{t}\right)_{t}$ represent the solutions of the $T_{c}$ equation (25) obtained for a negative value of the product of the uncoupled singlet and triplet $T_{c}$ equations on the left-hand side of equation (25). Therefore, $T_{c}\left(\Delta_{s}, \Delta_{t}\right)_{s}$ and $T_{c}\left(\Delta_{s}, \Delta_{t}\right)_{t}$ lie between the critical 


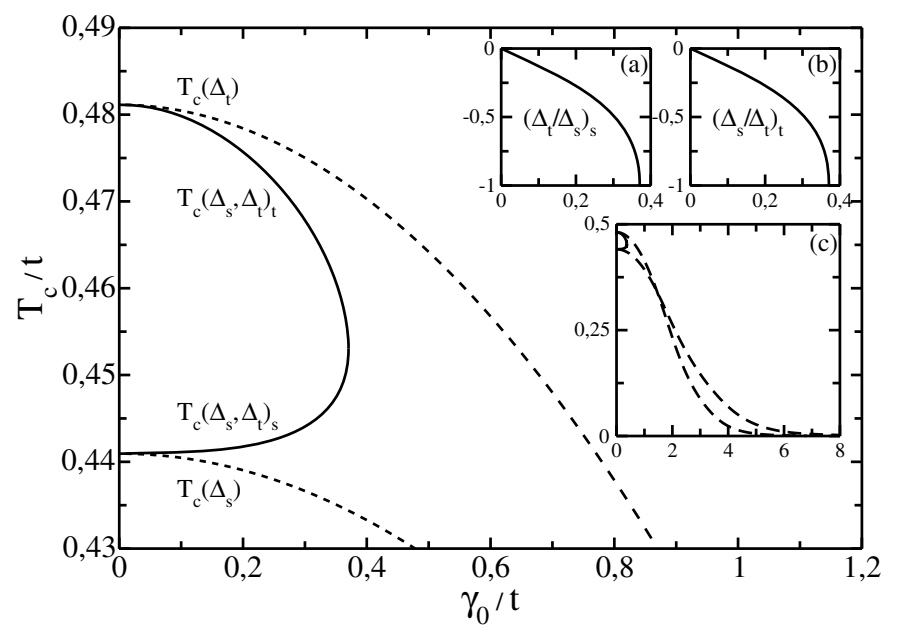

Fig. 8. Critical temperature of the coupled $s$-wave and $p$-wave state (solid line), triplet state (upper dashed-line) and the singlet state (lower dashed-line) for the pair potentials in the singlet and triplet channels $V_{s} / t=3, V_{t} / t=3$, and the band filling $n=1.2$. Insets: Relative magnitude of the subdominant and dominant components for $\boldsymbol{d}(\boldsymbol{k})$ antiparallel to $\gamma(\boldsymbol{k})$ vs. the spin-orbit coupling strength $\gamma_{0} / t$ for the dominant (a) $s$-wave state, (b) $p$-wave state. (c) Phase diagram for the entire range of the spin-orbit coupling rate.

temperatures $T_{c}\left(\Delta_{s}\right)$ of the singlet state and $T_{c}\left(\Delta_{t}\right)$ of the triplet state. The above constraint on $T_{c}\left(\Delta_{s}, \Delta_{t}\right)_{s}$ and $T_{c}\left(\Delta_{s}, \Delta_{t}\right)_{t}$ implies the enhancement of the lower and reduction of the higher of them by the increasing spin-orbit coupling rate. As a result a possible onset of the coupled singlet-triplet state for the nearly degenerate singlet and triplet states is limited to a very narrow range of the spinorbit coupling. Such a very weak pairing in the mixed singlet-triplet channel is manifested for equal pair potential amplitudes in the singlet and triplet channels. We present this issue for $V_{s} / t=V_{t} / t=3$ in Figure 8. The relative magnitude of the triplet and singlet components is determined by a solution of either (23) or (24) and reads

$$
\frac{\Delta_{t}}{\Delta_{s}}= \pm \frac{1-V_{s} \sum_{\boldsymbol{k}} e^{2}(\boldsymbol{k}) f_{+}\left(\varepsilon_{\boldsymbol{k}}\right)}{V_{s} \sum_{\boldsymbol{k}} e(\boldsymbol{k}) d(\boldsymbol{k}) f_{-}\left(\varepsilon_{\boldsymbol{k}}\right)}
$$

where the upper (lower) sign corresponds to $\boldsymbol{d}(\boldsymbol{k})$ parallel (antiparallel) to $\gamma(\boldsymbol{k})$.

When the critical temperatures of the dominant states $T_{c}\left(\Delta_{s}\right), T_{c}\left(\Delta_{t}\right)$ significantly differ the mixed state critical temperatures $T_{c}\left(\Delta_{s}, \Delta_{t}\right)_{s}$ and $T_{c}\left(\Delta_{s}, \Delta_{t}\right)_{t}$ remain close to the critical temperatures of the singlet and triplet states $T_{c}\left(\Delta_{s}\right), T_{c}\left(\Delta_{t}\right)$ for a wide range of the spin-orbit coupling rate. In consequence, there is a wide range of existence of the mixed states. We present this type of the singlet-triplet coupling in Figure 9 for the intermediate-coupling singlet pairing $\left(V_{s} / t=3\right)$ and the weak-coupling triplet pairing $\left(V_{t} / t=1\right)$. Comparison of Figures 8 and 9 shows that the relative magnitude of the singlet and triplet counterparts changes its sign for exchanged critical temperatures

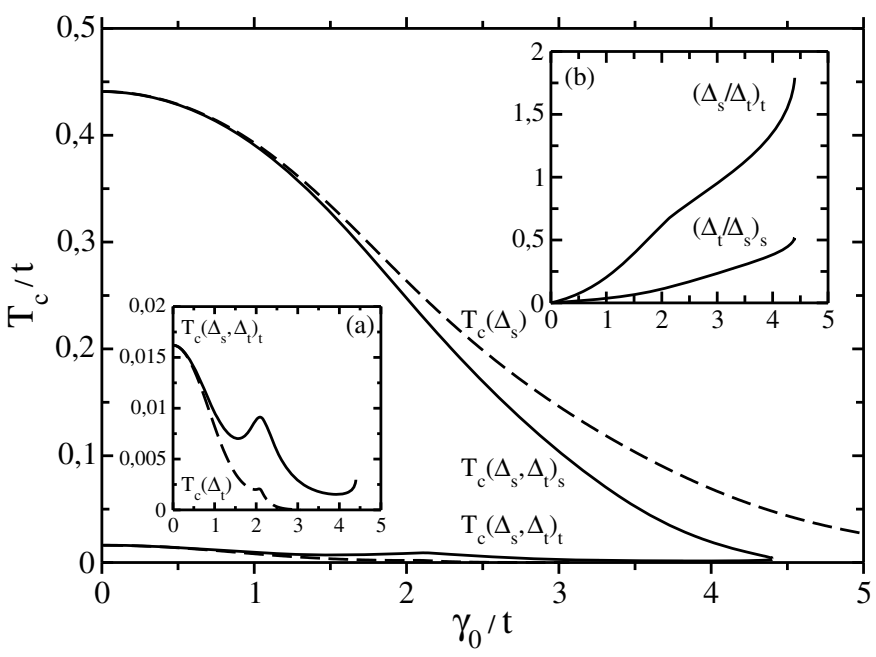

Fig. 9. Critical temperature of a coupled $s$-wave and $p$-wave state (solid line), singlet state (upper dashed-line) and the triplet state (lower dashed-line) for the pair potentials in the singlet and triplet channels $V_{s} / t=3, V_{t} / t=1$, and the band filling $n=1.2$. Insets: (a) enlarged phase diagram of the mixed state (solid line) for the dominant triplet state (dashed-line), the maximum stems from the van Hove singularity at the Fermi level; (b) relative magnitude of the subdominant and dominant components for $\boldsymbol{d}(\boldsymbol{k})$ antiparallel to $\boldsymbol{\gamma}(\boldsymbol{k})$ vs. the spin-orbit coupling strength $\gamma_{0} / t$ for the dominant $s$-wave state $\left(\Delta_{t} / \Delta_{s}\right)_{s}$ and the dominant $p$-wave state $\left(\Delta_{s} / \Delta_{t}\right)_{t}$.

$T_{c}\left(\Delta_{s}\right), T_{c}\left(\Delta_{t}\right)$ of the dominant states. This property follows directly from equation (28) which changes sign from negative for Figure 8 to positive for Figure 9 . We note that, the evolution of the relative magnitude of the subdominat and dominant states displayed in Figures 8 and 9 can be confirmed by the approximate iterative solutions of equations (23) and (24). Taking the singlet counterpart given by equation (23) as the dominant one $\left(\Delta_{t}=0\right)$ we have the subdominant component determined by equation (24) and vice versa for the dominant triplet order parameter. This simple procedure leads to approximately identical relative magnitude of both components for equal pair potentials in the singlet and triplet channels (Fig. 8), and approximately three times larger $\left(\Delta_{s} / \Delta_{t}\right)_{t}$ than $\left(\Delta_{t} / \Delta_{s}\right)_{s}$ for $V_{s}=3 V_{t}$ (Fig. 9). In summary, we have presented a weakcoupling theory of the onset of the mixed singlet-triplet state in systems with broken inversion symmetry employing a phenomenological pair potential which preserves the symmetry of the superconducting state. Although our results are unlikely to be used in a quantitative analysis of the experimental data they manifest the symmetry determined fundamental properties of a non-centrosymmetric superconducting system. We indicate that our conclusion of the coupling of the singlet $A_{1}$ state and the triplet state determined by $\boldsymbol{d}(\boldsymbol{k})$ aligned with $\boldsymbol{\gamma}(\boldsymbol{k})$ agrees with a microscopic result of Takimoto and Thalmeier [25] who showed within the spin fluctuations pairing mechanism a possible development of a stable coupled state in $\mathrm{CePt}_{3} \mathrm{Si}$ by a singlet $A_{1}$ state and a triplet state satisfying a condition $|\boldsymbol{d}(\boldsymbol{k}) \cdot \gamma(\boldsymbol{k})|=|\boldsymbol{d}(\boldsymbol{k})||\gamma(\boldsymbol{k})|$. Although the triplet state 
discussed in reference [25] exhibits an additional momentum structure represented by an overall symmetry preserving momentum-dependent amplitude $\Phi(\boldsymbol{k})$ it reduces to $\boldsymbol{d}(\boldsymbol{k})$ parallel or antiparallel to $\gamma(\boldsymbol{k})$ for $\Phi(\boldsymbol{k})= \pm 1$. On the other hand, our result does not corresponds to the strong-coupling calculation [26] predicting the $d+f$ wave symmetry of the mixed singlet-triplet state in the non-centrosymmetric superconductors.

\section{Conclusion}

Concluding, we have studied the stability of the superconducting states in the presence of the antisymmetric spinorbit coupling including the effect of the induced change of the single-particle density of states which affects both the spin singlet and the spin triplet states. We emphasize that, although the quantitative discussion of the $s$-wave and $p$-wave superconductivity has been carried out for the nearest neighbor tight-binding model, the qualitative conclusions follow from two basic features representative to any non-centrosymmetric system - the spin-split singleparticle energy spectrum and a concomitant redistribution of the spectral weights in the density of states. We have established that in general the antisymmetric spinorbit coupling induced split of the energy band leads to a monotonic suppression of the critical temperature of both singlet and triplet states. However, a redistribution of the spectral weights may generate the $T_{c}$ maxima in a form of sharp or broad peaks in the limit of the weak- or intermediate-coupling superconductivity, respectively, for the spin-orbit coupling strengths which give rise to singularities or significant peaks of the density of states at the Fermi level. This possible variation of the $T_{c}$ dependence on the spin-orbit coupling rate is highly pronounced for the $s$-wave state and becomes significantly weaker for the $p$-wave states due to their nodal structure. In comparison to former studies [6], we have established that the commonly accepted result of a constant and spin-orbit coupling independent critical temperature of the $s$-wave and the $\boldsymbol{d}(\boldsymbol{k}) \| \boldsymbol{\gamma}(\boldsymbol{k}) p$-wave states holds approximately only when the spin-orbit coupling effect on the energy spectrum can be neglected. However, as we have shown on the example of the $A_{2}$ state in the half-filled nearest neighbor tight-binding system, the unaltered $T_{c}$ of the nodal states may be additionally sustained for a very narrow range of the pairing strength by a particular location in the Brillouin zone of the pair potential nodes with respect to the singular points of the Fermi surface density of states. Considering the intermediate-coupling triplet superconductivity we have indicated that the pair-breaking effect of the antisymmetric spin-orbit coupling on the interband $\left(A_{1}\right)$ and mixed inter- and intraband $\left(B_{1}, B_{2}\right)$ states is significantly reduced and results in a comparable suppression of the $B_{2}$ state and the intraband $A_{2}$ state for the weak spin-orbit coupling. Finally, we have discussed the mixed singlet-triplet superconducting instability and showed that the coupled singlet-triplet state can be formed by the singlet $A_{1}$ state and the triplet state determined by the $\boldsymbol{d}(\boldsymbol{k})$-vector which is not orthogonal to the spinorbit coupling vector $\gamma(\boldsymbol{k})$. We have established that the development of such a mixed state is limited to very low values of the spin-orbit coupling for nearly degenerate singlet and triplet states. For significantly disparate critical temperatures of the even and odd parity components the mixed singlet-triplet state can be formed for a wide range of the spin-orbit coupling rate determined by the stability of the singlet and triplet states.

Although we have limited our considerations to the two-dimensional superconductivity, we note that the spin-orbit coupling induced evolution of the spin-split energy band represents a fundamental feature of the non-centrosymmetric systems irrespective of their dimensionality. Therefore, we presume that the obtained $T_{c}$ suppression patterns, except for the dimension-dependent quantitative changes, apply also to the bulk systems. Nevertheless, we indicate that the two-dimensional superconductivity [27-29] in the presence of the antisymmetric spin-orbit coupling may be developed by the surface electrons which form two-dimensional metallic bands in metals [30-32] or are confined by the interface potential barrier like in the superconducting $\mathrm{LaAlO}_{3} / \mathrm{SrTiO}_{3}$ interface [33].

The evolution of the critical temperature with the rising level of the antisymmetric spin-orbit coupling can be realized experimentally by the appropriate atomic substitutions [34] and such a tuned spin-orbit coupling effect on the superconducting instability temperature may provide a tool for a preliminary identification of a superconducting state.

\section{Authors contribution statement}

Authors contributed equally to the paper.

We thank R. Micnas and P. Grzybowski for valuable comments and literature references. Work has been supported by the grant POIG.02.02. 00-003/08 Narodowe Laboratorium Technologii Kwantowych.

\section{References}

1. E. Bauer, G. Hilscher, H. Michor, C. Paul, E.W. Scheidt, A. Gribanov, Y. Seropegin, H. Noel, M. Sigrist, P. Rogl, Phys. Rev. Lett. 92, 027003 (2004)

2. Non-centrosymmetric Superconductors, Introduction and Overview, edited by E. Bauer, M. Sigrist, Vol. 847 of Lecture Notes in Physics (Springer, Heidelberg, 2012)

3. Y.A. Bychkov, E.I. Rashba, J. Exp. Theor. Phys. Lett. 39, 78 (1984)

4. V.M. Edelstein, Zh. Eksp. Teor. Fiz. 95, 2151 (1989)

5. L.P. Gor'kov, E.I. Rashba, Phys. Rev. Lett. 87, 037004 (2001)

6. P.A. Frigeri, D.F. Agterberg, A. Koga, M. Sigrist, Phys. Rev. Lett. 92, 097001 (2004)

7. V. Barzykin, L.P. Gor'kov, Phys. Rev. Lett. 89, 227002 (2002) 
8. E. Bauer, R.T. Khan, H. Michor, E. Royanian, A. Grytsiv, N. Melnychenko-Koblyuk, P. Roghl, D. Reith, R. Podloucky, E.W. Scheidt et al., Phys. Rev. B 80, 064504 (2009)

9. E. Bauer, G. Roghl, X.Q. Chen, R.T. Khan, H. Michor, G. Hilscher, E. Royanian, K. Kumagai, D.Z. Li, Y.Y. Li et al., Phys. Rev. B 82, 064511 (2010)

10. K.V. Samokhin, E.S. Zijlstra, S.K. Bose, Phys. Rev. B 69, 094514 (2004)

11. M.J. Winiarski, M. Samsel-Czekała, Intermetallics 56, 44 (2015)

12. E. Bauer, P. Rogl, Non-centrosymmetric Superconductors: Strong vs. Weak Electronic Correlations, Vol. 847 of Lecture Notes in Physics (2012), pp. 3-33

13. Y. Ōnuki, R. Settai, Electronic States and Superconducting Properties of Non-centrosymmetric Rare Earth Compounds, Vol. 847 of Lecture Notes in Physics (2012), pp. 81-126

14. A.A. Abrikosov, L.P. Gorkov, I.E. Dzyaloshinski, Methods of Quantum Field Theory in Statistical Physics (Dover Publications, INC., New York, 1975)

15. P.A. Frigeri, D.F. Agterberg, I. Milat, M. Sigrist, Eur. Phys. J. B 54, 435 (2006)

16. Y. Tada, N. Kawakami, S. Fujimoto, New J. Phys. 11, 055070 (2009)

17. T. Shibayama, M. Nohara, H.A. Katori, Y. Okamoto, Z. Hiori, H. Takagi, J. Phys. Soc. Jpn 76, 073708 (2007)

18. A. Harada, N. Tamura, H. Mukuda, Y. Kitaoka, K. Wakui, S. Akutagawa, J. Akimitsu, J. Phys. Soc. Jpn 78, 025003 (2009)

19. S. Fujimoto, Bulletin Phys. Soc. Jpn 63, 20 (2008)

20. Y. Yanase, M. Sigrist, J. Phys. Soc. Jpn 76, 124709 (2007)

21. S.C. Zhang, Phys. Rev. Lett. 65, 120 (1990)

22. R.T. Scalettar, N.E. Bickers, D.J. Scalapino, Phys. Rev. Lett. 62, 1407 (1989)
23. M. Sigrist, K. Ueda, Rev. Mod. Phys. 63, 239 (1991)

24. I.A. Sergienko, S.H. Curnoe, Phys. Rev. B 70, 214510 (2004)

25. T. Takimoto, P. Thalmeier, J. Phys. Soc. Jpn 78, 103703 (2009)

26. T. Yokoyama, S. Onari, Y. Tanaka, Phys. Rev. B 75, $172511(2007)$

27. Y. Levi, O. Millo, A. Sharoni, Y. Tsabba, G. Leitus, S. Reich, Europhys. Lett. 51, 564 (2000)

28. T. Nishio, T. An, A. Nomura, K. Miyachi, T. Eguchi, H. Sakata, S. Lin, N. Hayashi, N. Nakai, M. Machida et al., Phys. Rev. Lett. 101, 167001 (2008)

29. P. Das, C.V. Tomy, S.S. Banerjee, H. Takeya, S. Ramakrishnan, A.K. Grover, Phys. Rev. B 78, 214504 (2008)

30. S. LaShell, B.A. McDougall, E. Jensen, Phys. Rev. Lett. 77, 3419 (1996)

31. L. Petersen, P.T. Sprunger, P. Hofmann, E. Lægsgaard, B.G. Briner, M. Doering, H.P. Rust, A.M. Bradshaw, F. Besenbacher, E.W. Plummer, Phys. Rev. B 57, R6858 (1998)

32. E. Rotenberg, J.W. Chung, S.D. Kevan, Phys. Rev. Lett. 82, 4066 (1999)

33. N. Reyren, S. Thiel, A.D. Caviglia, L.F. Kourkoutis, G. Hammerl, C. Richter, C.W. Schneider, T. Kopp, A.S. Ruetschi, D. Jaccard et al., Science 317, 1196 (2007)

34. E. Bauer, Superconductivity in materials without inversion symmetry (Karpacz Winter School of Theoretical Physics, 2014)

Open Access This is an open access article distributed under the terms of the Creative Commons Attribution License (http://creativecommons.org/licenses/by/4.0), which permits unrestricted use, distribution, and reproduction in any medium, provided the original work is properly cited. 